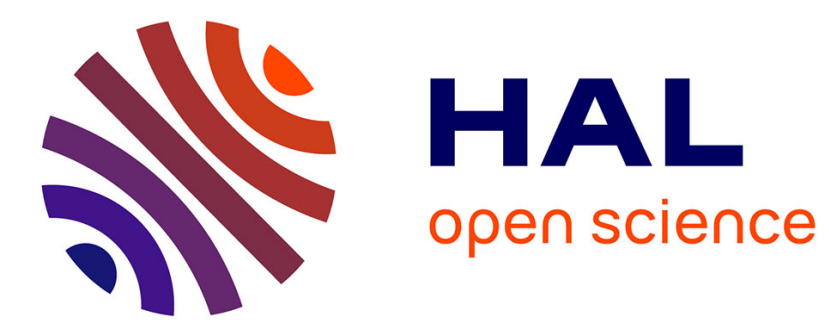

\title{
Stability Analysis for a Class of Linear 2x2 Hyperbolic PDEs Using a Backstepping Transform
}

\author{
David Bou Saba, Federico Bribiesca Argomedo, Jean Auriol, Michaël Di \\ Loreto, Florent Di Meglio
}

\section{To cite this version:}

David Bou Saba, Federico Bribiesca Argomedo, Jean Auriol, Michaël Di Loreto, Florent Di Meglio. Stability Analysis for a Class of Linear 2x2 Hyperbolic PDEs Using a Backstepping Transform. IEEE Transactions on Automatic Control, 2020, 65 (7), pp.2941 - 2956. 10.1109/TAC.2019.2934384 . hal02267587

\section{HAL Id: hal-02267587 \\ https://hal.science/hal-02267587}

Submitted on 19 Aug 2019

HAL is a multi-disciplinary open access archive for the deposit and dissemination of scientific research documents, whether they are published or not. The documents may come from teaching and research institutions in France or abroad, or from public or private research centers.
L'archive ouverte pluridisciplinaire HAL, est destinée au dépôt et à la diffusion de documents scientifiques de niveau recherche, publiés ou non, émanant des établissements d'enseignement et de recherche français ou étrangers, des laboratoires publics ou privés. 


\title{
Stability Analysis for a Class of Linear $2 \times 2$ Hyperbolic PDEs Using a Backstepping Transform
}

\author{
David Bou Saba ${ }^{1}$, Federico Bribiesca-Argomedo ${ }^{1}$, Jean Auriol ${ }^{2}$, Michael Di Loreto ${ }^{1}$, Florent Di Meglio ${ }^{2}$ \\ ${ }^{1}$ Univ Lyon, INSA Lyon, Laboratoire Ampère CNRS UMR5005, F-69621, Villeurbanne, France \\ ${ }^{2}$ MINES ParisTech, PSL Research University, CAS - Centre Automatique et Systèmes, 60 bd St Michel, 75006 Paris, France
}

\begin{abstract}
In this paper, we develop a sufficient stability condition for a class of coupled first-order linear hyperbolic PDEs with constant coefficients that appear when considering target systems for backstepping boundary control. Using a backstepping transform, the problem is reformulated as a stability problem for a difference equation with distributed delay. Finding the explicit solution to the backstepping kernels, we derive an explicit sufficient condition depending on the plant coefficients. This stability condition is compared to an existing stability result based on a Lyapunov analysis. Both the proposed and existing sufficient conditions are then contrasted in some examples to a (computationally expensive) numerical approximation of a necessary and sufficient condition for exponential stability to illustrate their conservatism.
\end{abstract}

Index Terms-Hyperbolic Partial Differential Equations, Difference equations, Stability analysis, Backstepping design.

\section{INTRODUCTION}

Systems of first-order hyperbolic PDEs, often representing systems of conservation and balance laws, have been broadly studied both from the control and analysis perspectives. They appear in many practically relevant engineering models, including those of electric transmission lines [1], hydraulic channels [2], pipelines [3]-[4], heat exchangers [5] and oil well drilling [6]. Different control approaches have been employed for the stabilization or observation of such systems (see for instance [6], [7], [8], [9], [10] and [11]). These control designs are accompanied by a stability analysis to ensure that the resulting closed-loop system (or, equivalently, the target system in the case of backstepping control) is stable. When in-domain and boundary couplings are both present (both of which can be sources of instability), the stability analysis of these systems can be challenging. Therefore, practical ways to guarantee their stability are required.

Some existing stability conditions for systems of conservation and balance laws (in different norms) can be found for instance in [12]. Other available results, based on (weighted $L^{2}$ ) Lyapunov functions, are [13] and [14] for the nonlinear and switched hyperbolic cases, respectively. The authors of [15] treat the case of symmetric hyperbolic PDEs, and prove the stability of these systems under the hypothesis of dissipation both inside the domain and through the boundary conditions. In [16], a sufficient stability condition was developped for

Corresponding author: M. Bou Saba (email: david.bou-saba@insa-lyon.fr). a coupled hyperbolic PDE-ODE system using a Lyapunov functional. Singular perturbation analysis was used in [17] for systems of conservation laws and in [18] for hyperbolic systems of balance laws. This method was explored in [19] and [20] for the development of sufficient stability conditions for systems of hyperbolic PDEs coupled to an ODE, and in [21] for the case of a wave equation coupled to an ODE. Also, in [22], Input-to-State Stability (ISS) bounds for hyperbolic PDEs are used to give sufficient conditions for stability of systems of two coupled hyperbolic PDEs in the sup norm.

Interestingly, systems of first-order hyperbolic PDEs can be transformed to neutral functional equations as in [23] or to a difference-delay system as in [24]. In [25], the authors established the equivalence between a single first-order hyperbolic PDE and systems described by an integral delay equation by proving that the mapping between the solutions is unique in appropriate spaces. Furthermore, it is easier to analyze the stability when converting the first-order hyperbolic PDEs to a delay form. In [26], a difference equation with distributed delay was derived from 2 coupled hyperbolic PDEs to achieve a robust stabilization with respect to input delays.

Given this relation between hyperbolic PDEs and delay systems it makes sense to also consider existing stability results for the latter. In the case of difference equations with pointwise and distributed delays, stability conditions can be found in [27] and [28], and, in the case of delay-differential equations in [29], [30], [31], [32] and [33].

The motivation for this work is to enlarge the class of target systems that can be considered when designing a backstepping controller. In the first available backstepping designs for these systems, boundary reflections in the target system were regarded as undesirable and were eliminated, together with some well-chosen in-domain coupling terms, allowing for a relatively simple stability analysis [9], [34], [35], [36]. Given that all of these systems were finite-time stable (and many were minimal-time convergent [37], [38]) the question of adding degrees of freedom to the controller was not extremely urgent to address. Nevertheless, recent results have made clear that removing reflections often results in closed-loop systems that have zero delay margins [39], [26]. Since it is now known that, in general, some reflections must be preserved, we end up with few target system options for which the stability analysis is simple. At the same time, any new target systems will naturally be exponentially stable instead of finite-time stable, 
which means that adding degrees of freedom that allow us to alter the rate of convergence and transient responses is more important than ever. It is within this context that we develop a new sufficient condition for the stability analysis of such target systems that is easy to verify and depends explicitly on the system coefficients.

It should be noted that this is not the first time alternate target systems have been studied. As an example, some recent works on boundary control of port-Hamiltonian systems, have also considered alternative internal coupling structures [40], [41], yet these designs rely on canceling boundary reflections and the use of specific strict Lyapunov functions for stability analysis, which impose a particular structure for the retained closed-loop system (it requires some degree of dissipation in the domain).

In this work, we analyze the stability of a class of $2 \times 2$ linear hyperbolic coupled PDEs. Using a backstepping transformation, the original system is mapped to a target system that can be written as a difference equation with distributed delay. This fact highlights the usefulness of the backstepping technique not only for control design, but for analysis as well. Furthermore, to the best of the authors' knowledge, few easily verifiable conditions exist for the class of systems obtained after this backstepping transform, which contain a distributed delay, and even then they would not be expressed in terms of the original system parameters, adding a supplementary layer of complexity to the analysis.

The contribution of this paper is the introduction of an easy to apply, explicit in terms of the plant parameters, sufficient condition for stability for a class of hyperbolic PDEs that can be naturally obtained through the backstepping procedure when applied to sytems of $2 \times 2$, counterconvecting, linear hyperbolic PDEs with constant coefficients.

In order to construct this result, we need to derive the explicit expressions of the backstepping kernels, using a similar procedure to that used in [42]. Incidentally, combining the results of [42] and the present paper yields analytic expressions of the complete set of backstepping kernels (both direct and inverse) which can be useful for analyzing transient behavior of the closed-loop system. These analytic expressions allow us to express the target system as a difference equation with distributed delay for which a necessary and sufficient stability condition can be found in the Laplace domain [43]. Upperbounding some of the terms in this necessary and sufficient condition we are able to derive a relatively simple sufficient stability condition, which is roughly equivalent (in terms of complexity) to that in [12, Theorem 5.4] for the same class of PDEs. These two explicit sufficient conditions obtained by completely different approaches are then compared and shown to not be equivalent, as neither one implies the other.

In order to illustrate the conservatism of the proposed condition, it is compared to a numerical approximation of the necessary and sufficient condition. This approximation is obtained by leveraging the explicit expressions of the backstepping kernels in order to both restrict the region of the complex plane where unstable poles may exist and then to determine a stopping criterion that, for stable systems and with a perfect computer, should result in a finite execution time. In practice, some tolerance is added in order to account for numerical errors and to avoid infinite execution times for unstable systems.

The paper is organized as follows. In Section II, we present the class of systems under consideration. In Section III, we reformulate the original system as a difference equation with distributed delay by means of a backstepping transform and we give the associated necessary and sufficient stability condition in the Laplace domain. In Section IV, an explicit sufficient stability condition in terms of the original system coefficients is proposed. This condition is compared to an existing (and similarly complex) condition obtained by Lyapunov analysis directly and through some examples. A numerical approximation of the necessary and sufficient condition is then constructed. In Section V, this numerical approximation is applied, together with both explicit conditions, to some illustrative examples to assess the conservatism of the proposed condition. Concluding remarks are given in Section VI.

Throughout this paper, we use the following definitions. We define the functional space $\chi \doteq L^{2}([0,1] ; \mathbb{R}) \times L^{2}([0,1] ; \mathbb{R})$, the space where the PDE states will be defined, with the associated norm

$$
\|(\varphi, \psi)\|_{\chi} \doteq\left(\int_{0}^{1} \varphi^{2}(\theta) \mathrm{d} \theta+\int_{0}^{1} \psi^{2}(\theta) \mathrm{d} \theta\right)^{1 / 2},
$$

for any $(\varphi, \psi) \in \chi$. We define as well the partial trajectory of a function $\varphi$ as $\varphi_{[t]}(\theta) \doteq \varphi(t+\theta), \theta \in[-\tau, 0)$, with norm in $L^{2}([-\tau, 0) ; \mathbb{R}), \tau>0$

$$
\left\|\varphi_{[t]}\right\|_{L^{2}([-\tau, 0) ; \mathbb{R})} \doteq\left(\int_{-\tau}^{0} \varphi^{2}(t+\theta) \mathrm{d} \theta\right)^{1 / 2}
$$

for $t \geq \tau$, and its norm in $C^{1}([-\tau, 0] ; \mathbb{R})$ will be defined as

$$
\left\|\varphi_{[t]}\right\|_{C^{1}([-\tau, 0] ; \mathbb{R})} \doteq \max _{\theta \in[-\tau, 0]}|\varphi(t+\theta)|+\max _{\theta \in[-\tau, 0]}\left|\varphi_{t}(t+\theta)\right|
$$

for $t \geq \tau . \partial_{t} \varphi$ will designate the partial derivative of $\varphi$ with respect to the independent variable $t$.

We define the Banach algebra $\mathcal{A}$ equipped with a standard convolution product in the sense of [43]. $\mathcal{A}$ consists of BIBOstable generalized functions of the form

$$
g(t)=g_{0}(t)+\sum_{i=1}^{\infty} g_{i} \delta\left(t-t_{i}\right)
$$

with $g_{0} \in L^{1}\left(\mathbb{R}^{+} ; \mathbb{R}\right)$ and $\sum_{i=1}^{\infty}\left|g_{i}\right|<\infty$. $\delta$ denotes the unit Dirac distribution. The algebra $\mathcal{A}$ is equipped with the norm

$$
\|g\|_{\mathcal{A}} \doteq\left\|g_{0}\right\|_{L^{1}}+\sum_{i=1}^{\infty}\left|g_{i}\right| \text {. }
$$

We denote $\hat{\mathcal{A}}$ the set of Laplace transforms of elements in $\mathcal{A} . \hat{\mathcal{A}}$ is a Banach algebra with unit. The norm on $\hat{\mathcal{A}}$ satisfies $\|\hat{g}\|_{\hat{\mathcal{A}}}=\|g\|_{\mathcal{A}}$, where $\hat{g}$ stands for the Laplace transform of $g \in \mathcal{A}$.

$J_{n}$ and $I_{n}$, for $n \in \mathbb{Z}$, stand for the Bessel functions and the modified Bessel functions of the first kind, respectively. 
Given a set $\Omega \subseteq \mathbb{R}$, its characteristic function $\mathbb{1}_{\Omega}: \mathbb{R} \rightarrow \mathbb{R}$ is defined as

$$
\mathbb{1}_{\Omega}(\theta) \doteq \begin{cases}1 & \text { if } \theta \in \Omega \\ 0 & \text { otherwise. }\end{cases}
$$

A function $f$ defined on $\omega \subseteq \mathbb{C}$ is Lipschitz-continuous with Lipschitz constant $m$ on $\omega$, if there exists a constant $m \geq 0$ such that $\left|f\left(z_{1}\right)-f\left(z_{2}\right)\right| \leq m\left|z_{1}-z_{2}\right|, \forall z_{1}, z_{2} \in \omega$, where $|\cdot|$ denotes the modulus of an element in $\mathbb{C}$.

\section{Problem StATEMEnT}

We are interested in the stability analysis of a class of $2 \times$ 2 linear first-order hyperbolic coupled PDEs. Systems under consideration are of the form

$$
\begin{aligned}
\partial_{t} u(t, x)+\frac{1}{r_{1}} \partial_{x} u(t, x) & =\sigma_{1} v(t, x) \\
\partial_{t} v(t, x)-\frac{1}{r_{2}} \partial_{x} v(t, x) & =\sigma_{2} u(t, x)
\end{aligned}
$$

for all $t \geq 0, x \in[0,1]$, where $\frac{1}{r_{1}}>0$ and $\frac{1}{r_{2}}>0$ are the transport velocities associated to the two transport equations (6) and (7) respectively. The coefficients $\sigma_{1}$ and $\sigma_{2}$ represent constant in-domain couplings between the PDEs. The associated initial conditions are

$$
\begin{aligned}
& u_{0}(\cdot) \doteq u(0, \cdot) \\
& v_{0}(\cdot) \doteq v(0, \cdot),
\end{aligned}
$$

defined in $L^{2}([0,1], \mathbb{R})$. In addition, $u(t, x)$ and $v(t, x)$ satisfy the following boundary conditions

$$
\begin{aligned}
& u(t, 0)=q v(t, 0) \\
& v(t, 1)=\rho u(t, 1)
\end{aligned}
$$

for all $t>0$, where $\rho$ and $q$ are boundary coupling coefficients such that

$$
|\rho q|<1
$$

Note that if $|\rho q| \geq 1$, the system cannot be stable [12, Theorem 2.6]. We assume that $\rho$ and $q$ are not both zero. Without loss of generality, we consider that $q \neq 0$.

The reader will notice first that no diagonal coupling terms are considered in (6)-(7). This is partly because these terms can be removed using an exponential transform together with the backstepping control design presented in [9], [10], and therefore such a target system is always achievable (when considering constant-coefficient equations), and also because, if kept, they prevent the development of the explicit expressions for the backstepping kernels. One could also attempt to use only the exponential change of variables such as that in [11], [44] in order to absorb the diagonal coupling terms into the off-diagonal ones at the expense of non-constant coefficients, which pose the same problem. Therefore we only consider the case where diagonal coefficients either do not appear in the original system or, more likely, are compensated by a backstepping-based control.

Our goal is therefore to construct sufficient stability conditions that guarantee the exponential stability of system (6)-(9) in an $L^{2}$ sense. That is, for any $\left(u_{0}, v_{0}\right) \in \chi$, there exist $\mu>0$ and $C \geq 0$, such that the solution $(u, v)$ of system (6)-(9) satisfies

$$
\|(u(t, \cdot), v(t, \cdot))\|_{\chi} \leq C\left\|\left(u_{0}, v_{0}\right)\right\|_{\chi} \mathrm{e}^{-\mu t}, \quad t \geq 0 .
$$

As a first step, we will employ a backstepping transform that leads to a target system where the in-domain couplings are shifted to the boundary. The structure of the target system will then allow us to reformulate the system as a difference equation with distributed delay.

\section{DIFFERENCE EQUATION WITH DISTRIBUTED DELAY}

In this section, we consider the class of PDEs described by (6)-(9) and establish an equivalent realization as a difference equation. The objective of this section is to give a necessary and sufficient stability condition of the system. To do so, we use a backstepping transform, then the Laplace transform and therefore analyze the BIBO stability [43] in Laplace domain.

\section{A. Backstepping transform}

In order to rewrite the original system in a difference equation form, we employ the following Volterra transformation used in [10]

$$
\begin{aligned}
u(t, x)= & \alpha(t, x)-\int_{0}^{x} L^{11}(x, y) \alpha(t, y) \mathrm{d} y \\
& -\int_{0}^{x} L^{12}(x, y) \beta(t, y) \mathrm{d} y \\
v(t, x)= & \beta(t, x)-\int_{0}^{x} L^{21}(x, y) \alpha(t, y) \mathrm{d} y \\
& -\int_{0}^{x} L^{22}(x, y) \beta(t, y) \mathrm{d} y,
\end{aligned}
$$

where the kernels $L^{i j}, i, j \in\{1,2\}$ verify the following PDEs in the triangular domain $\mathcal{T} \doteq\{(x, y) \in[0,1] \times[0,1], y \leq$ $x\}$ [10]

$$
\begin{aligned}
& \frac{1}{r_{1}} \partial_{x} L^{11}(x, y)+\frac{1}{r_{1}} \partial_{y} L^{11}(x, y)=\sigma_{1} L^{21}(x, y) \\
& \frac{1}{r_{1}} \partial_{x} L^{12}(x, y)-\frac{1}{r_{2}} \partial_{y} L^{12}(x, y)=\sigma_{1} L^{22}(x, y) \\
& \frac{1}{r_{2}} \partial_{x} L^{21}(x, y)-\frac{1}{r_{1}} \partial_{y} L^{21}(x, y)=-\sigma_{2} L^{11}(x, y) \\
& \frac{1}{r_{2}} \partial_{x} L^{22}(x, y)+\frac{1}{r_{2}} \partial_{y} L^{22}(x, y)=-\sigma_{2} L^{12}(x, y),
\end{aligned}
$$

with boundary conditions

$$
\begin{aligned}
L^{12}(x, x) & =-\sigma_{1} \frac{r_{1} r_{2}}{r} \\
L^{11}(x, 0) & =L^{12}(x, 0) \frac{r_{1}}{q r_{2}} \\
L^{21}(x, x) & =\sigma_{2} \frac{r_{1} r_{2}}{r} \\
L^{22}(x, 0) & =L^{21}(x, 0) \frac{r_{2}}{r_{1}} q,
\end{aligned}
$$

where we denote $r \doteq r_{1}+r_{2}$.

Given $q \neq 0$, equations (13)-(14) are of the class considered in [10, Theorem A.2]. Accordingly, these equations have a unique solution $L^{i j}, i, j \in\{1,2\}$ in $C^{\infty}(\mathcal{T} ; \mathbb{R})$. 
To the best of the authors' knowledge, an analytic solution for these equations is not given in the literature, including [10]. Since an analytic solution is needed in our analysis, we develop in Appendix A the explicit expression of the backstepping kernels in terms of Bessel functions.

Lemma 1: PDEs (13) with boundary conditions (14), have a unique smooth solution, given by (63)-(66).

The proof is given in Appendix A.

We consider the following target system

$$
\begin{aligned}
& \partial_{t} \alpha(t, x)+\frac{1}{r_{1}} \partial_{x} \alpha(t, x)=0 \\
& \partial_{t} \beta(t, x)-\frac{1}{r_{2}} \partial_{x} \beta(t, x)=0,
\end{aligned}
$$

with initial conditions

$$
\begin{aligned}
& \alpha_{0}(\cdot) \doteq \alpha(0, \cdot) \\
& \beta_{0}(\cdot) \doteq \beta(0, \cdot),
\end{aligned}
$$

defined in $L^{2}([0,1], \mathbb{R})$, and boundary conditions

$$
\begin{aligned}
\alpha(t, 0)= & q \beta(t, 0) \\
\beta(t, 1)= & \rho \alpha(t, 1)+\int_{0}^{1} N^{\alpha}(y) \alpha(t, y) \mathrm{d} y \\
& +\int_{0}^{1} N^{\beta}(y) \beta(t, y) \mathrm{d} y,
\end{aligned}
$$

where

$$
\begin{aligned}
& N^{\alpha}(y) \doteq L^{21}(1, y)-\rho L^{11}(1, y) \\
& N^{\beta}(y) \doteq L^{22}(1, y)-\rho L^{12}(1, y) .
\end{aligned}
$$

The backstepping transform (12) is a bounded linear operator from $\chi$ to $\chi[10]$, and maps the target system (15)-(18) to the original system (6)-(9). Notice that the backstepping transform (12) is an inverse transform, i.e., it explicitly maps the target system to the original system. It is used instead of the direct transform since it allows us to write the boundary condition (18) as function of the states $\alpha$ and $\beta$.

Proposition 2: For any initial condition $\left(\alpha_{0}, \beta_{0}\right) \in$ $\chi$, the target system (15)-(18) has a unique weak solution $(\alpha(t, \cdot), \beta(t, \cdot)) \in \chi$, for $t \geq 0$ and $\beta_{[t]}(\cdot, 1) \in$ $L^{2}([-r, 0) ; \mathbb{R}), \forall t \geq r$.

Proof: An initial condition $\left(\alpha_{0}, \beta_{0}\right) \in \chi$ is mapped, by the backstepping transform (12), to an initial condition $\left(u_{0}, v_{0}\right) \in \chi$. According to [12, Theorem A.4], the Cauchy problem (6)-(9) with this initial condition has a unique solution $(u(t, \cdot), v(t, \cdot)) \in \chi$. Since the transform (12) is a boundedly invertible operator from $\chi$ to $\chi$, we conclude the proof.

Remark 1: Assume that $u_{0}$ and $v_{0} \in C^{1}([0,1] ; \mathbb{R})$, and the initial conditions of the original system verify the following compatibility conditions

$$
\begin{aligned}
& u_{0}(0)=q v_{0}(0) \\
& v_{0}(1)=\rho u_{0}(1) . \\
& u_{0}^{\prime}(0)=-q \frac{r_{1}}{r_{2}} v_{0}^{\prime}(0)-q r_{1} \sigma_{2} u_{0}(0)+r_{1} \sigma_{1} v_{0}(0) \\
& v_{0}^{\prime}(1)=-\rho \frac{r_{2}}{r_{1}} u_{0}^{\prime}(1)-r_{2} \sigma_{2} u_{0}(1)+\rho r_{2} \sigma_{1} v_{0}(1) .
\end{aligned}
$$

Adapting the proof of $\left[10\right.$, Theorem A.1] to $C^{1}([0,1] ; \mathbb{R})$, we have that the target system (15) has a unique solution $\alpha(t, x)$ and $\beta(t, x)$ verifying $\alpha(t, \cdot)$ and $\beta(t, \cdot) \in C^{1}([0,1] ; \mathbb{R}), \forall t \geq 0$ and $\beta_{[t]}(\cdot, 1) \in C^{1}([-r, 0] ; \mathbb{R}), \forall t \geq r$.

\section{B. Distributed delay form}

Following the method of characteristics, the transport equations (15) can be written as the following difference equations

$$
\begin{aligned}
& \alpha(t, x)=\alpha\left(t-r_{1} x, 0\right), \quad \forall t \geq r_{1} x \\
& \beta(t, x)=\beta\left(t-r_{2}(1-x), 1\right), \quad \forall t \geq r_{2}(1-x)
\end{aligned}
$$

$\forall x \in[0,1]$. Plugging equations (21)-(22) in the terms of the boundary condition (18) verified by $\beta(t, 1)$ and using (17) yield

$$
\begin{aligned}
\alpha(t, 1) & =\alpha\left(t-r_{1}, 0\right) \\
& =q \beta(t-r, 1), \\
\int_{0}^{1} N^{\alpha}(y) \alpha(t, y) \mathrm{d} y & =q \int_{0}^{1} N^{\alpha}(y) \beta\left(t-r_{2}-r_{1} y, 1\right) \mathrm{d} y \\
& =\frac{q}{r_{1}} \int_{r_{2}}^{r} N^{\alpha}\left(\frac{\theta-r_{2}}{r_{1}}\right) \beta(t-\theta, 1) \mathrm{d} \theta, \\
\int_{0}^{1} N^{\beta}(y) \beta(t, y) \mathrm{d} y & =\int_{0}^{1} N^{\beta}(y) \beta\left(t-r_{2}(1-y), 1\right) \mathrm{d} y \\
& =\frac{1}{r_{2}} \int_{0}^{r_{2}} N^{\beta}\left(1-\frac{\theta}{r_{2}}\right) \beta(t-\theta, 1) \mathrm{d} \theta .
\end{aligned}
$$

Finally, the difference equation with distributed delay satisfied by $\beta(t, 1)$ is

$$
\beta(t, 1)=\rho q \beta(t-r, 1)+\int_{0}^{r} N(\theta) \beta(t-\theta, 1) \mathrm{d} \theta
$$

for $t \geq r$, with

$$
\begin{aligned}
N(\theta)= & \frac{1}{r_{2}} N^{\beta}\left(1-\frac{\theta}{r_{2}}\right) \mathbb{1}_{\left[0, r_{2}\right]}(\theta) \\
& +\frac{q}{r_{1}} N^{\alpha}\left(\frac{\theta-r_{2}}{r_{1}}\right) \mathbb{1}_{\left[r_{2}, r\right]}(\theta), \theta \in[0, r] .
\end{aligned}
$$

Notice that $N^{\alpha}$ and $N^{\beta} \in C^{\infty}([0,1] ; \mathbb{R})$ (as defined in (19)) and $N \in C^{\infty}([0, r] ; \mathbb{R}) \subset L^{1}([0, r] ; \mathbb{R})$ (as defined in (25)). $N(\theta)$ can be expressed in terms of the Bessel functions (see Appendix A, equation (69)). The continuity of $N$ at $r_{2}$ is ensured due to the same expression satisfied by $N(\theta)$ for $\theta \in\left[0, r_{2}\right]$ (equation (67)) and $\theta \in\left(r_{2}, r\right]$ (equation (68)).

Equations (21)-(22) represent the solution to the PDE states inside the domain as a function of the solution at the boundaries.

One can check that the stability of $\beta(\cdot, 1)$ implies in particular the stability of the state $\beta$ satisfying (22). In turn, the stability of the state $\alpha$ is ensured at $x=0$ by (17) and inside the domain by (21). Furthermore, due to the boundedness and the invertibility of the backstepping transfrom, there is an equivalence between the stability of the original and the target systems. 
We also note that for $t \in[0, r]$, the regularity of the solution of the target system (15)-(18) depends on the regularity of the initial conditions $\alpha_{0}$ and $\beta_{0}$. Thus, since the transform (12) is boundedly invertible from $\chi$ to $\chi$ [10], any initial condition $\left(u_{0}, v_{0}\right) \in \chi$ is mapped to an initial condition $\left(\alpha_{0}, \beta_{0}\right) \in \chi$. Therefore, $(\alpha(t, \cdot), \beta(t, \cdot)) \in \chi$ for $t \in[0, r]$.

Consequently, we will focus our analysis on the boundary stability criterion and give stability conditions for $\beta(\cdot, 1)$ satisfying the difference equation with distributed delay (24).

\section{Necessary and sufficient stability condition}

We now present a necessary and sufficient stability condition for our system rewritten as a difference equation with distributed delay. Using the Laplace transform, the characteristic equation associated to (24) is

$$
\hat{\phi}(s) \doteq 1-\rho q \mathrm{e}^{-s r}-\int_{0}^{r} N(\theta) \mathrm{e}^{-s \theta} \mathrm{d} \theta=0 .
$$

For the stability analysis, we restrict our study to the halfplane $\operatorname{Re}(s) \geq 0$. Consider the transfer function

$$
\hat{G}(s) \doteq \frac{-\int_{0}^{r} N(\theta) \mathrm{e}^{-s \theta} \mathrm{d} \theta}{1-\rho q \mathrm{e}^{-s r}} .
$$

With $|\rho q|<1$, one can verify that the conditions required by [43, Theorem 1] are fulfilled by $\hat{G}$ allowing us to apply the stability result therein. In addition, we have that

$$
\hat{F}(s) \doteq \frac{\hat{G}(s)}{1+\hat{G}(s)}=\frac{-\int_{0}^{r} N(\theta) \mathrm{e}^{-s \theta} \mathrm{d} \theta}{\hat{\phi}(s)}
$$

and

$$
|1+\hat{G}(s)|=\frac{|\hat{\phi}(s)|}{\left|1-\rho q \mathrm{e}^{-s r}\right|} .
$$

Since $N(\cdot) \in C^{\infty}([0, r] ; \mathbb{R}), \int_{0}^{r} N(\theta) \mathrm{e}^{-s \theta} \mathrm{d} \theta$ is a Finite Impulse Response (FIR) filter, the stability of $\hat{F}(s)$ is equivalent to the absence of roots of $\hat{\phi}(s)$ in the complex right half-plane. According to [43, Theorem 1], $\hat{\phi}$ has an inverse in $\hat{\mathcal{A}}$ if and only if

$$
\inf _{\operatorname{Re}(s) \geq 0}|1+\hat{G}(s)|>0,
$$

which, by (29), is equivalent to

$$
\inf _{\operatorname{Re}(s) \geq 0}|\hat{\phi}(s)|>0 .
$$

Condition (31) is necessary and sufficient for the exponential stability of $\beta(\cdot, 1)$ satisfying $(24)$ in $L^{2}((-r, 0] ; \mathbb{R})$ according to $\left[45\right.$, Remark 1], provided that $\left(\alpha_{0}, \beta_{0}\right) \in \chi$. The stability of system (6)-(9) in the $\chi$-norm is consequently equivalent to (31).

This necessary and sufficient condition cannot be easily verified since it requires the evaluation of $|\hat{\phi}|$ at every point $s$ in the complex right half-plane (RHP). This evaluation is complicated by the distributed delay term (integral term in (26)) and, therefore, it is not a practical tool to check the stability of the system under consideration.

However, one may proceed by upper-bounding the modulus of the terms in $\hat{\phi}$. This yields a simpler (only sufficient) condition, that is much easier to apply and depends only on the system coefficients. This is the purpose of Section IV-A.

Another approach to tackle this problem would be to numerically approximate this condition. This is done in Section IV-C for validation purposes. Despite being more computationally expensive, the numerical approximation is less conservative than the explicit criterion. Crucially for the implementation of the algorithm, we only need to verify condition (31) in a compact domain in the RHP which can be analytically bounded in terms of the system coefficients, as will appear in Section IV-C.

Both alternatives require some sort of solution of $N(\theta)$. It is possible to get the backstepping gains without an explicit expression, by using numerical successive approximations. Nevertheless, applying this method is more complicated than using their analytic expressions and could yield more conservative criteria since approximation errors have to be taken into account. Instead, we explicitly solve the hyperbolic PDEs (13)(14) and obtain the resulting expression of $N(\theta)$. This is essential in order to state an explicit stability criterion and to construct the compact domain where the numerical algorithm makes the tests. In addition, it is used to derive the expressions of many parameters required by the algorithm (as will be detailed in section IV-C). Appendix A contains the derivations of the explicit solution to the hyperbolic PDEs satisfied by the backstepping kernel gains, leading to the expressions of $N(\theta), N^{\prime}(\theta)$ and the upper-bounds of their absolute values.

\section{STABILITY ANALYSIS OF A DIFFERENCE EQUATION WITH DISTRIBUTED DELAY: SUFFICIENT CONDITIONS}

In this section, we present the explicit sufficient stability condition for the system under consideration, obtained by relaxing the necessary and sufficient condition previosly presented. This explicit criterion, in terms of the system coefficients is compared to a similarly complex criterion available in the literature and, by numerically approximating the necessary and sufficient condition, an algorithm is constructed. Its task is to verify the validity of (31) inside a compact domain in the RHP.

\section{A. Explicit sufficient condition}

We state now a sufficient stability condition of the system under consideration, depending on the sign of $\sigma_{1} \sigma_{2}$ and $\rho q$. In order to simplify the notation, we define the parameters

$$
\begin{aligned}
a \doteq q r_{2} \sigma_{2}+\rho r_{1} \sigma_{1} \\
R \doteq r_{1} r_{2} \sigma_{1} \sigma_{2} .
\end{aligned}
$$

Proposition 3: If the constant parameters of system (6)-(9) satisfy either of the following set of inequalities:

(i)

$$
\begin{gathered}
\sigma_{1} \sigma_{2} \geq 0, \quad \rho q \geq 0 \text { and } \\
|a|+|R|\left(\frac{1}{1+|\rho q|}-\frac{1-|\rho q|}{2}\right)<1-|\rho q|, \\
\sigma_{1} \sigma_{2} \geq 0, \quad \rho q<0 \quad \text { and } \\
|a|+|R| \frac{1+|\rho q|}{2}<1-|\rho q|,
\end{gathered}
$$


(iii)

$$
\begin{aligned}
& \sigma_{1} \sigma_{2}<0, \quad \rho q \geq 0 \text { and } \\
& |a| I_{0}(\sqrt{|R|})+|R|\left(\frac{1}{1+|\rho q|}-\frac{1-|\rho q|}{2}\right) \\
& \times\left[I_{0}(\sqrt{|R|})-I_{2}(\sqrt{|R|})\right]<1-|\rho q|,
\end{aligned}
$$

(iv)

$$
\begin{aligned}
& \sigma_{1} \sigma_{2}<0, \quad \rho q<0 \quad \text { and } \\
& |a| I_{0}(\sqrt{|R|})+|R| \frac{1+|\rho q|}{2} \\
& \times\left[I_{0}(\sqrt{|R|})-I_{2}(\sqrt{|R|})\right]<1-|\rho q|,
\end{aligned}
$$

then, system (6)-(9) is exponentially stable in the $\chi$-norm in the sense of (11).

Proof: In this proof, we rely on the necessary and sufficient condition (31), which is fulfilled if

$$
\sup _{\operatorname{Re}(s) \geq 0}|1-\hat{\phi}(s)|<1 .
$$

$|1-\hat{\phi}(s)|$ can be bounded as

$$
|1-\hat{\phi}(s)|=\left|\rho q \mathrm{e}^{-s r}+\int_{0}^{r} N(\theta) \mathrm{e}^{-s \theta} \mathrm{d} \theta\right| \leq|\rho q|+\int_{0}^{r}|N(\theta)| \mathrm{d} \theta .
$$

Using (35), a sufficient condition for (34) is

$$
\int_{0}^{r}|N(\theta)| \mathrm{d} \theta<1-|\rho q| .
$$

The crucial point is then the upper-bound of $\int_{0}^{r}|N(\theta)| \mathrm{d} \theta$ given in (84) in Appendix A, depending on the sign of $\sigma_{1} \sigma_{2}$ and $\rho q$. Substituting these expressions into (36), we conclude the proof.

Remark that inequality (36) can be tested by numerically approximating the integral term and using the expression of $N(\theta)$ in (69). In the case of spatially varying coefficients $\sigma_{1}(x)$ and $\sigma_{2}(x)$, (36) can also be tested, with $N(\theta)$ generated numerically using the successive approximation method.

Following the assumptions of Remark 1, and according to [45, Remark 1], the sufficient condition of Proposition 3 implies the point-wise exponential stability of system (24). In other words, there exist $\mu>0$ and $C \geq 0$ such that

$$
|\beta(t, 1)| \leq C \sup _{-r \leq \theta \leq 0}|\beta(\theta, 1)| \mathrm{e}^{-\mu(t-r)}, \quad t \geq r .
$$

Let us now compare the presented result to some existing results in the literature, and apply it to some illustrative examples.

\section{B. Comparison of the explicit sufficient condition with other criteria and numerical validation}

In order to evaluate the conservatism of the sufficient condition in Proposition 3, we compare it with other sufficient

\begin{tabular}{|c|c|c|}
\hline case & $\begin{array}{l}\text { Condition required by Proposition } \\
\qquad 3 \text { satisfied if }\end{array}$ & $\begin{array}{c}\text { Condition (39) } \\
\text { required by [12, } \\
\text { Theorem 5.4] } \\
\text { satisfied if }\end{array}$ \\
\hline $\begin{array}{c}\sigma_{1} \sigma_{2}>0 \text { or } \\
\text { only one of } \\
\text { them is equal } \\
\text { to zero }\end{array}$ & $\begin{array}{l}\frac{\rho q \geq 0}{} \\
\quad|a|+|R|\left(\frac{1}{1+|\rho q|}-\frac{1-|\rho q|}{2}\right)< \\
------------ \\
\frac{\rho q<0}{1-} \\
\quad|a|+|R| \frac{1+|\rho q|}{2}<1-|\rho q|\end{array}$ & (Not satisfied) \\
\hline $\begin{array}{l}\sigma_{1}=0 \\
\sigma_{2}=0\end{array}$ & $|\rho q|<1$ & $|\rho q|<1$ \\
\hline$\sigma_{1} \sigma_{2}<0$ & 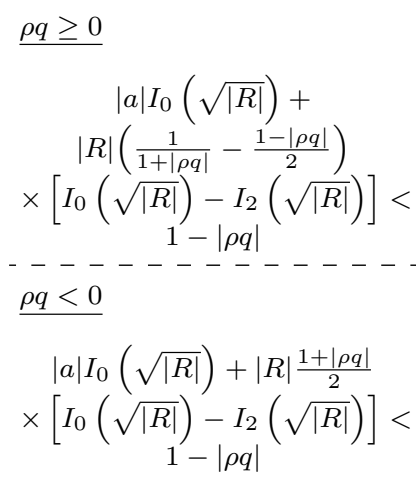 & $\rho^{2}<-\frac{\sigma_{2}}{\sigma_{1}} \frac{r_{2}}{r_{1}}<\frac{1}{q^{2}}$ \\
\hline
\end{tabular}
conditions from the literature for the stability of systems of linear first-order hyperbolic PDEs. In particular, we compare it with a Lyapunov-based exponential stability condition for linear systems of balance laws presented in [12] and an ISS and small-gain based result in [22].
Table I

COMPARISON BETWEEN THE CONDITION OF PROPOSITION 3 AND CONDITION (39) REQUIRED BY [12, THEOREM 5.4] FOR THE STABILITY OF SYSTEM (6)-(9)

According to [12, Theorem 5.4], system (6)-(9) (with constant coefficients $\sigma_{1}$ and $\sigma_{2}$ ) is exponentially stable in the $L^{2}$-norm if there exists a $2 \times 2$ positive diagonal real matrix $\Pi \doteq\left[\begin{array}{cc}\pi_{1} & 0 \\ 0 & \pi_{2}\end{array}\right]$ such that
$\Sigma^{\top} \Pi+\Pi \Sigma \succeq 0$
$\|P\|_{2}<1$

where $\Sigma=\left[\begin{array}{cc}0 & -\sigma_{1} \\ -\sigma_{2} & 0\end{array}\right]$ and $P=\left[\begin{array}{cc}0 & q \sqrt{\frac{\pi_{1}}{\pi_{2}} \frac{r_{2}}{r_{1}}} \\ \rho \sqrt{\frac{\pi_{2}}{\pi_{1}} \frac{r_{1}}{r_{2}}} & 0\end{array}\right]$. The norm considered in (38) is the spectral-norm, defined as the square root of the largest eigenvalue of $P^{\top} P$. This condition was originally derived for systems with diagonal coupling terms, possibly spatially varying. In case of (6)-(9), condition (38) reduces to: either $\sigma_{1}=\sigma_{2}=0$, or

$$
\begin{aligned}
& \sigma_{1} \sigma_{2}<0, \text { and } \\
& \qquad \rho^{2}<-\frac{\sigma_{2}}{\sigma_{1}} \frac{r_{2}}{r_{1}}<\frac{1}{q^{2}} .
\end{aligned}
$$

Otherwise, if $\sigma_{1} \sigma_{2}>0$ or only one of them is equal to zero, no conclusion can be made regarding the stability. We present in Table I the sufficient conditions required by Proposition 3 and [12, Theorem 5.4] for the different signs of $\sigma_{1} \sigma_{2}$ and $\rho q$.

One may check that in the case of $\sigma_{1}=\sigma_{2}=0$, system (6)(9) is viewed as a system of two linear conservation laws with two reflection terms at the boundaries. In the case of $\sigma_{1} \sigma_{2}<$ 0 , the condition of Proposition 3 and (39) are both sufficient 
Table II

VALIDATING EXAMPLES. All THESE SySTEMS ARE STABLE

\begin{tabular}{|c|c|c|}
\hline $\begin{array}{c}\text { Example of system (6)-(9) } \\
\left(\sigma_{1}, \sigma_{2}, r_{1}, r_{2}, \rho, q\right)\end{array}$ & $\begin{array}{c}\text { Condition of } \\
\text { Proposition 3 }\end{array}$ & $\begin{array}{c}\text { Condition (39) } \\
\text { required by [12, } \\
\text { Theorem 5.4] }\end{array}$ \\
\hline $\begin{array}{c}(1.1,0.4,1,1.2,0.4,-0.5) \\
(-0.8,0.7,1,1.2,0.4,0.25)\end{array}$ & Satisfied & $\begin{array}{c}\text { Not satisfied } \\
\left(\sigma_{1} \sigma_{2}>0\right)\end{array}$ \\
\hline $\begin{array}{c}(1.3,-0.95,1.8,0.44, \\
0.45,0.25)\end{array}$ & Satisfied & Satisfied \\
\hline $\begin{array}{c}(1.3,-1.2,1.8,1.5, \\
0.45,0.25)\end{array}$ & Not satisfied & Satisfied \\
\hline $\begin{array}{c}(2.3,-3.5,0.8,1.1, \\
0.5,-0.7)\end{array}$ & Not satisfied & Not satisfied \\
\hline
\end{tabular}

and can be applied simultaneously. For the sake of clarity, we give some numerical examples in Table II.

Another criterion for the stability of symmetric hyperbolic PDEs is found in [15]. When applying [15, Theorem 2.1] for the class of $2 \times 2$ constant-coefficients, the required condition is

$$
\begin{aligned}
& \sigma_{1}+\sigma_{2}=0, \text { and } \\
& \qquad c_{1} r_{2}+\rho^{2} \leq \frac{r_{2}}{r_{1}} \leq \frac{1}{q^{2}}-c_{2} \frac{r_{2}}{q^{2}},
\end{aligned}
$$

with $c_{1} \geq 0$ and $c_{2} \geq 0$ and $c_{1}$ and $c_{2}$ are not both zero. This criterion, developed under the hypothesis of a dissipation on both equation and the boundary is more restrictive than [12, Theorem 5.4] regarding the stability of the system with respect to the values of $\sigma_{1}$ and $\sigma_{2}$.

Using recent ISS results and a small-gain property, another sufficient stability condition for a related class of systems of hyperbolic PDEs is given in Chapter 9 of [22]. When applied to system (6)-(9), this condition requires the existence of a constant $K>0$, with $(|\rho|+|q|) e^{-K}<1$, such that

$$
\begin{aligned}
& \left(\sqrt{\frac{e^{2 K}-e^{K}}{K} r_{2}\left|\sigma_{2}\right|}+\sqrt{|\rho|}\right) \\
& \times\left(\sqrt{\frac{e^{2 K}-e^{K}}{K} r_{1}\left|\sigma_{1}\right|}+\sqrt{|q|}\right)<1 .
\end{aligned}
$$

This condition is not satisfied for the examples considered in Table II so an extra column was not added. It will however be compared to the numerical algorithm developed in the next section.

As we can see from these results, the stability of some systems can be tested using the condition required by Proposition 3. Nevertheless, many other cases are still not covered, i.e. we cannot conclude regarding the stability of many plants by applying both sufficient criteria. The conservatism in our approach is a result of bounding the modulus of the exponential terms in $\hat{\phi}$ in (35). An alternative is to consider the necessary and sufficient condition and numerically approximate it. That will be the goal of the next section, where an algorithm is constructed and reveals to be less conservative than both approaches previously compared at a much higher computational cost.
C. Numerical approximation of the Necessary and Sufficient condition

We develop a numerical test of the condition (31) for the stability of system (24), based on the explicit expressions of $N(\theta), N^{\prime}(\theta)$ and the upper-bounds of their absolute values, given in Appendix A. Our objective is to test the absence of zeros (up to a certain precision) in the right half plane of the characteristic equation (26).

Notice that numerical algorithms for spectrum analysis for time-delay systems as DELAY_PSA developed in [46], YALTA in [47] or DDE-BIFTOOL in [48] require explicit characteristic quasipolynomials. However, such analytic representation can not be handled in our problem, and we are restricted to use only a numerical realization for the distributed delay. For this, a numerical algorithm for (pseudo)spectrum of the time-delay plant (24) is developed in this section.

This study requires the consideration of the whole RHP. However, having the explicit expression of $N(\theta)$ and therefore the behavior of $\hat{\phi}$, we can restrict our analysis to a compact subset of the RHP, which is the only subset of the RHP where equation (26) can have roots as detailed by Lemma 4 . This will allow us to construct a finite initial grid for the evaluation of $\hat{\phi}$ which we will then refine as needed. Using the Lipschitz constant of $\hat{\phi}$ the algorithm can conclude on the absence of roots in a region around each evaluation point. Whenever the evaluation of $\hat{\phi}$ is insufficient to guarantee the absence of roots between two adjacent evaluation points, the evaluation grid is refined around those points. This operation is repeated until the algorithm can either guarantee the absence of roots in the initial compact set (in which case we conclude that the system is stable) or a certain tolerance level in the grid size or value of $|\hat{\phi}|$ is reached (in which case we cannot conclude on the stability of the system).

Let the compact subset of $\mathbb{C}$ :

$\mathcal{C} \doteq\left\{s=z+i y \in \mathbb{C}\right.$ s.t. $0 \leq z \leq z_{l}$ and $\left.|s| \leq M(z)\right\}$, where we define

$$
\begin{aligned}
z_{l} & \doteq \frac{|\rho q|}{r}+\nu \\
M(z) & \doteq \frac{|N(r)| \mathrm{e}^{-z r}+|N(0)|+\frac{\bar{\nu}}{z}\left(1-\mathrm{e}^{-z r}\right)}{1-|\rho q| \mathrm{e}^{-z r}},
\end{aligned}
$$

with

$$
\begin{aligned}
& N(0)=\frac{1}{r}(a+R) \\
& N(r)=\frac{1}{r}(a-\rho q R) .
\end{aligned}
$$

The values of $a$ and $R$ are given in (32) and (33) respectively. $\nu$ and $\bar{\nu}$, given in (82), are the upper-bounds of $|N(\theta)|$ and $\left|N^{\prime}(\theta)\right|$ respectively.

An illustration of such a compact is shown in Figure 1. The parameters chosen for this example are $\sigma_{1}=2, \sigma_{2}=$ $1.5, r_{1}=0.85, r_{2}=1.1, \rho=0.7$ and $q=-0.4$.

Lemma 4: By definition of $\mathcal{C}$, we have that

$$
\exists \epsilon>0, \text { such that } \forall s \in \operatorname{RHP} \backslash \mathcal{C},|\hat{\phi}(s)| \geq \epsilon .
$$




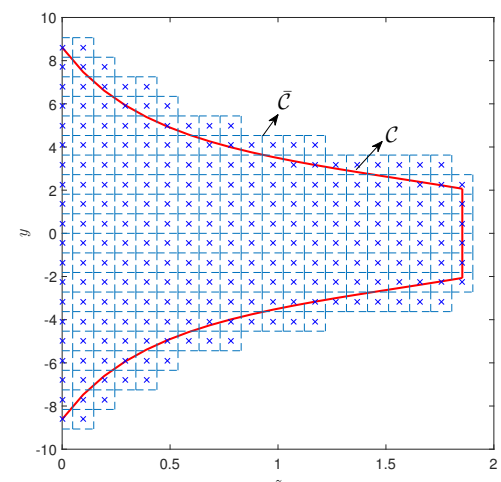

Figure 1. Grid $\mathcal{G}^{0}$ construction inside $\overline{\mathcal{C}} \supset \mathcal{C}$

Proof: $z>z_{l}$ yields

(44)

$$
\begin{aligned}
& |\hat{\phi}(s)|=\left|1-\rho q \mathrm{e}^{-s r}-\int_{0}^{r} N(\theta) \mathrm{e}^{-s \theta} \mathrm{d} \theta\right| \\
& \geq 1-\left|\rho q \mathrm{e}^{-s r}\right|-\left|\int_{0}^{r} N(\theta) \mathrm{e}^{-s \theta} \mathrm{d} \theta\right| \\
& \quad \geq 1-|\rho q| \mathrm{e}^{-z r}-\nu \int_{0}^{r} \mathrm{e}^{-z \theta} \mathrm{d} \theta \\
& \quad \geq 1-|\rho q| \mathrm{e}^{-z r}-\frac{\nu}{z}+\frac{\nu}{z} \mathrm{e}^{-z r} \\
& \quad \geq 1-|\rho q| \mathrm{e}^{-z r}-\frac{\nu}{z} \\
& \quad \geq 1-\frac{|\rho q|}{z r}-\frac{\nu}{z} \\
& \quad=1-\frac{z_{l}}{z}>0 .
\end{aligned}
$$

On the other hand, if $|s|>M(z)$, and by integrating by parts the term $\int_{0}^{r} N(\theta) \mathrm{e}^{-s \theta} \mathrm{d} \theta$ to get

$$
\begin{aligned}
\int_{0}^{r} N(\theta) \mathrm{e}^{-s \theta} \mathrm{d} \theta= & -\frac{N(r)}{s} \mathrm{e}^{-s r}+\frac{N(0)}{s} \\
& +\frac{1}{s} \int_{0}^{r} N^{\prime}(\theta) \mathrm{e}^{-s \theta} \mathrm{d} \theta
\end{aligned}
$$

we have

$$
\begin{aligned}
& |\hat{\phi}(s)| \geq 1-|\rho q| \mathrm{e}^{-z r}-\frac{|N(r)| \mathrm{e}^{-z r}}{|s|}-\frac{|N(0)|}{|s|}-\frac{\bar{\nu}}{z|s|}\left(1-\mathrm{e}^{-z r}\right) \\
& \geq 1-\frac{M(z)}{|s|}>0 .
\end{aligned}
$$

Note that $M(z)$ is strictly decreasing with the increase of $z$ and $\lim _{z \rightarrow 0} M(z)=\frac{|N(r)|+|N(0)|+\bar{\nu} r}{1-|\rho q|}$ and $\lim _{z \rightarrow \infty} M(z)=|N(0)|$.

Let us first construct a grid defined as a set $\mathcal{G}^{0}$ of nodes in a compact $\overline{\mathcal{C}}$ containing $\mathcal{C}$ as depicted in Figure 1 . The nodes in the grid are equally distant along the $z$ and $y$ axis with steps denoted by $\delta z$ and $\delta y$ respectively.

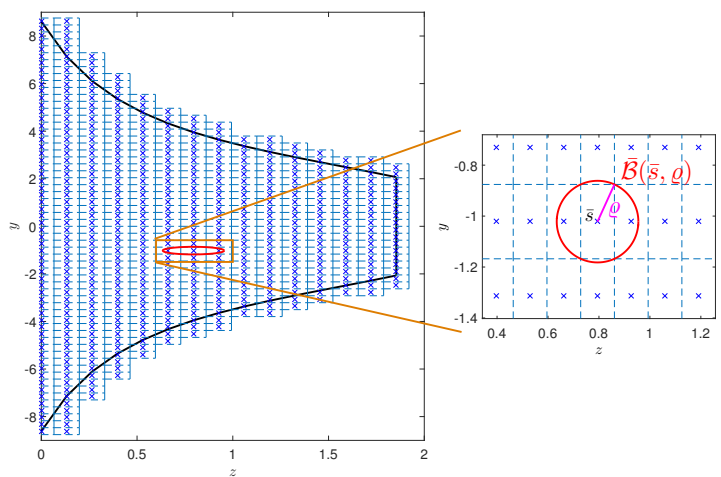

Figure 2. Closed ball $\overline{\mathcal{B}}(\bar{s}, \varrho)$

In order to cover all the compact subset $\overline{\mathcal{C}}$ by means of a finite number of nodes, we consider $\overline{\mathcal{B}}(s, \varrho)$ consisting in a closed ball centered at each $s \in \mathcal{G}^{0}$ and having a radius

$$
\varrho \doteq \frac{1}{2} \sqrt{\delta z^{2}+\delta y^{2}}
$$

as shown in Figure 2. Notice that $\mathcal{C} \subset \overline{\mathcal{C}} \subset \cup_{s \in \mathcal{G}^{0}} \overline{\mathcal{B}}(s, \varrho)$. The algorithm is based on the following Lemma.

Lemma 5: Let the parameter

$$
\kappa \doteq r|\rho q|+\frac{1}{2} r^{2} \nu
$$

If at a certain point $\bar{s} \in \mathcal{G}^{0}$,

$$
|\hat{\phi}(\bar{s})|>\kappa \varrho,
$$

then $|\hat{\phi}(s)|>0 \forall s \in \overline{\mathcal{B}}(\bar{s}, \varrho)$.

Proof: having $N \in C^{\infty}([0, r] ; \mathbb{R}), \hat{\phi}$ is Lipschitzcontinuous. Furthermore

$$
\begin{aligned}
\| \hat{\phi}\left(s_{1}\right)|-| \hat{\phi}\left(s_{2}\right)|| & \leq\left|\hat{\phi}\left(s_{1}\right)-\hat{\phi}\left(s_{2}\right)\right| \\
& \leq \max _{s \in \overline{\mathcal{C}}}\left\{\left|\frac{\partial}{\partial z} \hat{\phi}(s)\right|,\left|\frac{\partial}{\partial y} \hat{\phi}(s)\right|\right\}\left|s_{1}-s_{2}\right|,
\end{aligned}
$$

with

$$
\begin{aligned}
\frac{\partial}{\partial y} \hat{\phi}(s) & =i \frac{\partial}{\partial z} \hat{\phi}(s) \\
& =i r \rho q \mathrm{e}^{-s r}+i \int_{0}^{r} \theta N(\theta) \mathrm{e}^{-s \theta} \mathrm{d} \theta
\end{aligned}
$$

which makes $|\hat{\phi}|$ Lipschitz-continuous with Lipschitz constant upper-bounded by $\kappa$.

In the light of this fact, the algorithm will start by calculating $|\hat{\phi}(s)| \forall s \in \mathcal{G}^{0}$. If $\forall s \in \mathcal{G}^{0}$, (49) is satisfied, then $|\hat{\phi}(s)|>0 \forall s \in \overline{\mathcal{C}}$. Consequently, $|\hat{\phi}(s)|>0 \forall \operatorname{Re}(s) \geq$ 0 and the system is stable according to the equivalent condition (31). Otherwise, a more detailed grid will be constructed by considering further nodes in the vicinity of every point $s \in \mathcal{G}^{0}$ where (49) is not verified (starting by the point where $|\hat{\phi}|$ has the lowest value) and by dividing the radius of the closed ball in (47) by 2 . We will denote a 


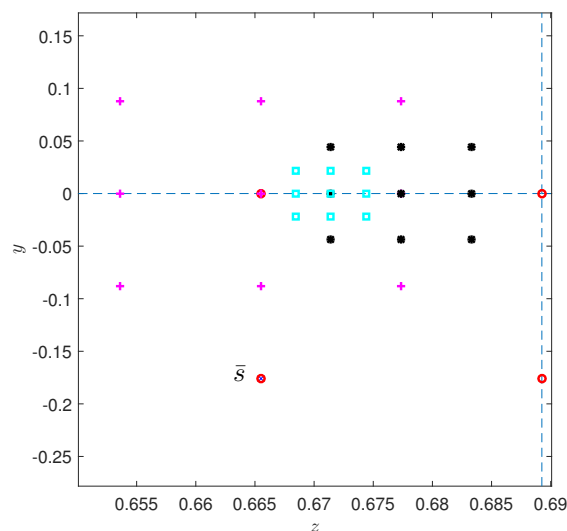

Figure 3. Example grid after the fourth iteration of the algorithm.

refined grid obtained at the vicinity of a point $\bar{s} \doteq \bar{z}+i \bar{y} \in \overline{\mathcal{C}}$ after $k$ recursive iterations by

$$
\begin{aligned}
\mathcal{G}^{k}(\bar{s}) \doteq\{s=z+i y \in \overline{\mathcal{C}} \quad \text { s.t. } \quad z & \in\left\{\bar{z}-\frac{\delta z}{2^{k}}, \bar{z}, \bar{z}+\frac{\delta z}{2^{k}}\right\} \\
\text { and } \quad y & \left.\in\left\{\bar{y}-\frac{\delta y}{2^{k}}, \bar{y}, \bar{y}+\frac{\delta y}{2^{k}}\right\}\right\} .
\end{aligned}
$$

In a refined grid $\mathcal{G}^{k},|\hat{\phi}|$ is calculated at all the newly considered nodes and a test similar to (49) will be executed. Before visiting another node in the original grid $\mathcal{G}^{0}$, the algorithm will track all the nodes in a refined grid using a similar technique of a depth-first search.

The algorithm will backtrack a node by moving to a higherlevel grid $\mathcal{G}^{k-1}$ if all the nodes of $\mathcal{G}^{k}$ verify

$$
|\hat{\phi}(s)|>\kappa \frac{\varrho}{2^{k}} .
$$

The iteration progress is shown in Figure 3. First, we consider a point $\bar{s}$ in $\mathcal{G}^{0}$ (blue ' $\times$ '), such that $|\hat{\phi}(\bar{s})|$ does not satisfy (49). Subsequently, $\mathcal{G}^{1}(\bar{s})$ is constructed (red circles). If the lowest value of $|\hat{\phi}|$ in $\mathcal{G}^{1}(\bar{s})$ does not satisfy (53) (for $k=1$ ), then $\mathcal{G}^{2}(\overline{\bar{s}})$ is constructed by adding more nodes in the vicinity of $\overline{\bar{s}} \doteq \operatorname{argmin}_{s \in \mathcal{G}^{1}(\bar{s})}|\hat{\phi}(s)|$ as shown in pink ' + ' signs. The algorithm will iterate in the same way to get the nodes pointed in black asterisks and cyan squares in subsequent steps.

The construction of a refined grid $\mathcal{G}^{k}$ will always be priority to the node $\bar{s}=\operatorname{argmin}_{s \in \mathcal{G}^{k-1}}|\hat{\phi}(s)|$ before backtracking the other nodes, in order to reduce the total time needed for a complete test in the case of unstable system.

The same procedure is repeated until one of the situations below occurs:

- The algorithm could visit all the points considered in $\mathcal{G}^{0}$ (and all the refined grids) and either the inequality (49) or (53) is satisfied for all the created nodes. The system is therefore stable.
- The process is still iterating at a certain point, by exploring more detailed grids until $|\hat{\phi}|$ reaches a tolerance level $\varepsilon$ (a predefined value), then the algorithm will return that there is no guarantee for the stability of the system.

A description of the algorithm is detailed in Algorithm 1.

Remark 2: Let us notice that the stability analysis of (24) can be carried out using the Nyquist stability criterion. Indeed, the associated characteristic equation (26) is equivalent to $1+$ $\hat{G}(s)=0$, where $\hat{G}(s)$ is defined in (27). This last equality corresponds to the characteristic equation of a feedback system whose open-loop transfer function is $\hat{G}(s)$, which is stable and strictly proper. In order to get stability for this feedback system, it is required that the Nyquist graph of $\hat{G}(i \omega)$ does not encircle -1 . Since $\hat{G}(s)$ is strictly proper, the graph of $\hat{G}(i \omega)$ has to be generated only over the finite frequency interval $\omega \in\left[0, \omega_{\max }\right]$ where $\omega_{\max }$ is such that $|\hat{G}(i \omega)|<1$ for all $\omega \geq \omega_{\max }$.

The construction of this frequency interval can be obtained as follows. From (45), we have, for all $\omega>0$,

$$
|\hat{N}(i \omega)| \leq \frac{|N(0)|+|N(r)|+\bar{\nu} r}{\omega},
$$

where $N(0)$ and $N(r)$ are defined in (43) while $\bar{\nu}$ is defined in (82). Introducing

$$
\omega_{\max }=\frac{|N(0)|+|N(r)|+\bar{\nu} r}{1-|\rho q|},
$$

we have that, for all $\omega>\omega_{\max },|\hat{N}(i \omega)|<1-|\rho q|$, which in turn implies $|\hat{G}(i \omega)|<1$ from (27). The Nyquist graph of $\hat{G}(i \omega)$ requires a numerical approximation of the distributed delay, that can be performed using standard techniques [49]. This algorithm leads to a simpler numerical test of stability than the one developed in Section IV-C, that can be used to test stability or instability with reduced complexity and a graphical criterion. The algorithm developed in Section IV-C uses instead analytical properties of the characteristic equation for testing stability or instability, which also points to the numerical location of (possible) poles of the plant in the righthalf complex plane.

We would like to emphasize the importance of the derivation of the kernel expressions in the Appendix A as they provide the possibility of having the explicit criterion and constructing the algorithm.

\section{Simulation Results}

In order to illustrate the validity of the algorithm, let us take the example of system (6)-(9) with the following parameters: $\sigma_{1}=2.3, \sigma_{2}=-3.5, r_{1}=0.8, r_{2}=1.1, \rho=0.5$ and $q=-0.7$. One can check that sufficient conditions suggested in Proposition 3, [12, Theorem 5.4] and [22] fail to prove the stability of this system. Meanwhile, the algorithm described above is able to visit all the nodes and successfully verify the criterion (53) as depicted in Figure 4.

Furthermore, let us compare the stability domains obtained by the explicit criterion of Proposition 3, condition (39) required by [12, Theorem 5.4], condition (41) obtained in [22] and the numerical algorithm. We take the following 

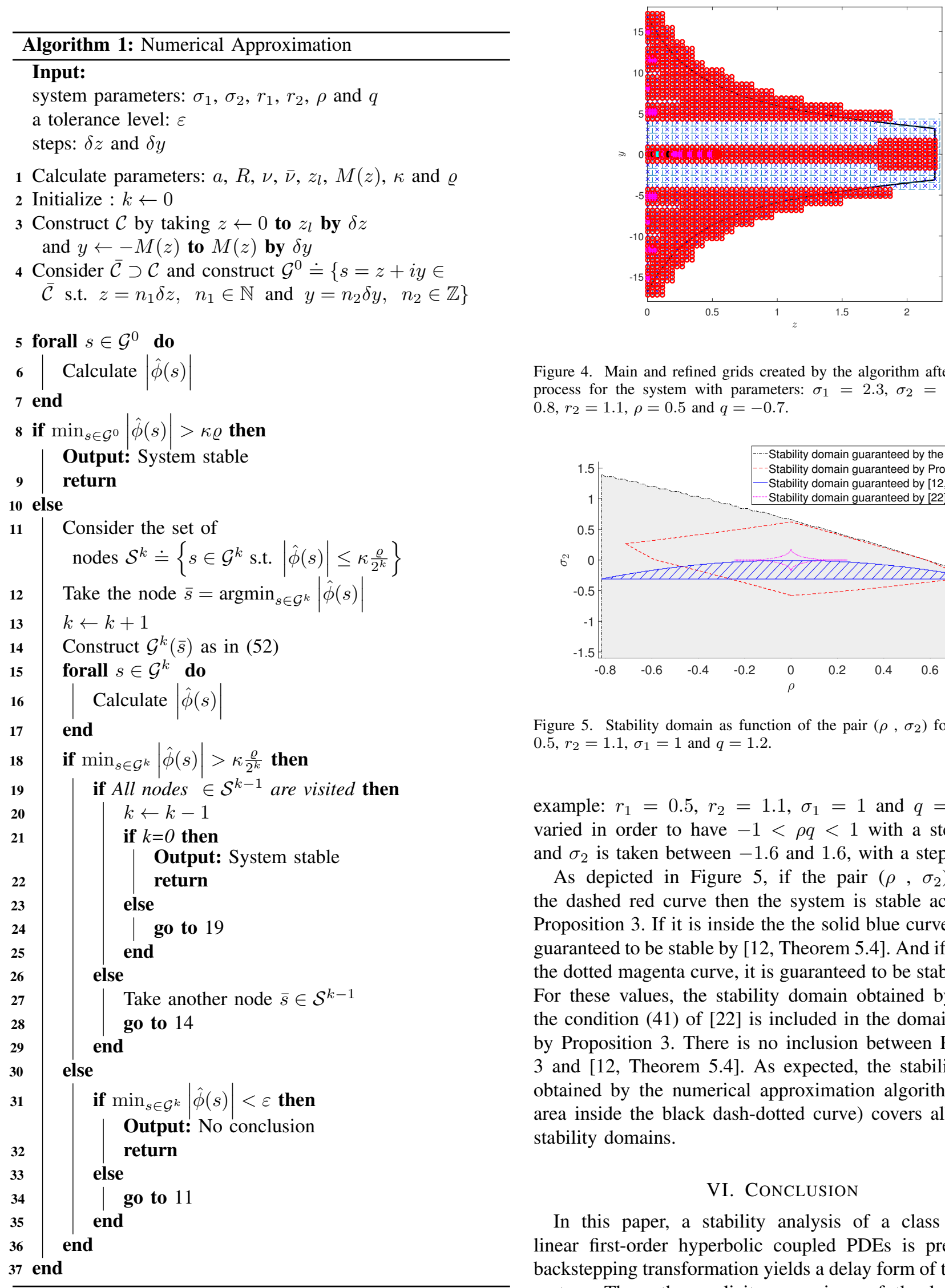

Figure 4. Main and refined grids created by the algorithm after a complete process for the system with parameters: $\sigma_{1}=2.3, \sigma_{2}=-3.5, r_{1}=$ $0.8, r_{2}=1.1, \rho=0.5$ and $q=-0.7$.

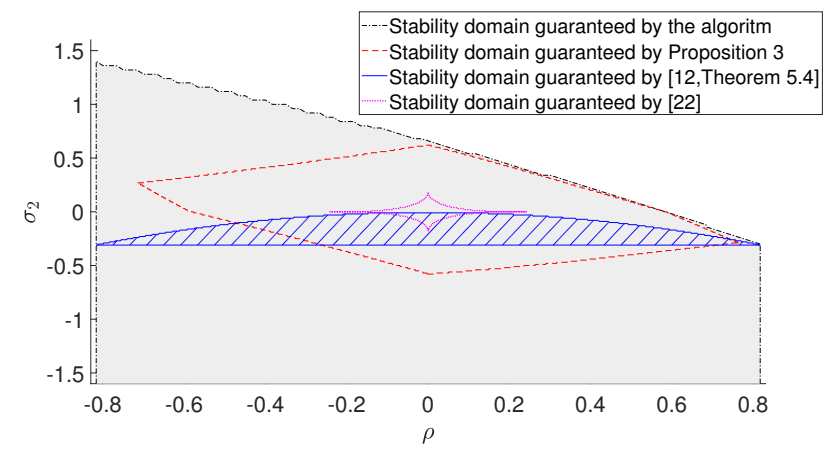

Figure 5. Stability domain as function of the pair $\left(\rho, \sigma_{2}\right)$ for fixed $r_{1}=$ $0.5, r_{2}=1.1, \sigma_{1}=1$ and $q=1.2$.

example: $r_{1}=0.5, r_{2}=1.1, \sigma_{1}=1$ and $q=1.2 . \rho$ is varied in order to have $-1<\rho q<1$ with a step of 0.02 and $\sigma_{2}$ is taken between -1.6 and 1.6, with a step of 0.04 .

As depicted in Figure 5, if the pair $\left(\rho, \sigma_{2}\right)$ is inside the dashed red curve then the system is stable according to Proposition 3. If it is inside the the solid blue curve, then it is guaranteed to be stable by [12, Theorem 5.4]. And if it is inside the dotted magenta curve, it is guaranteed to be stable by [22] For these values, the stability domain obtained by applying the condition (41) of [22] is included in the domain obtained by Proposition 3. There is no inclusion between Proposition 3 and [12, Theorem 5.4]. As expected, the stability domain obtained by the numerical approximation algorithm (shaded area inside the black dash-dotted curve) covers all the other stability domains.

\section{CONCLUSION}

In this paper, a stability analysis of a class of $2 \times 2$ linear first-order hyperbolic coupled PDEs is presented. A backstepping transformation yields a delay form of the original system. Then, the explicit expressions of the kernel gains of the backstepping transform are derived using the Bessel functions of the first kind. These expressions are used to obtain 
an explicit stability criterion in terms of the constant system coefficients, depending on their sign. The criterion is validated through academic examples. A numerical algorithm that guarantees the stability of the system in a less conservative way is developed. It works by testing if the necessary and sufficient condition is satisfied in a compact space in the right half plane. Interestingly, if we could deal with perfect computers, the algorithm would guarantee the stability with a finite number of steps. Future works will be focused on extending these results to general cases of linear first-order hyperbolic coupled PDEs, for instance, by taking into consideration diagonal in-domain couplings.

\section{ACKNOWLEDGMENTS}

The authors would like to thank the reviewers for their particularly insightful and thorough comments that had an important impact in improving the paper.

\section{APPENDIX A}

\section{PROOF OF LEMMA 1}

We derive the exact analytic solution for the kernel gains $L^{i j}, i, j \in\{1,2\}$ satisfying the hyperbolic PDEs (13) with boundary conditions (14), as functions of the constant parameters $\left(\sigma_{1}, \sigma_{2}, r_{1}, r_{2}, \rho\right.$ and $\left.q\right)$ of the original system (6)(9). Notice that the solution given in [42] using the Bessel and Marcum $Q$-functions cannot be applied since the class of systems considered is a $2 \times 2$ coupled hyperbolic PDEs, in contrast to the $4 \times 4$ systems that appear in (13)-(14).

We first find the integral equations satisfied by $L^{i j}$ before applying the successive approximation method that leads to power series expressions of these gains. Bessel functions and modified Bessel functions of the first kind are then used to give their exact analytic solution. Next, we find the analytic expressions of $N(\theta)$ and $N^{\prime}(\theta)$ and give upper-bounds of their absolute values. These solutions, as well as the upper-bounds, will be used to express the sufficient condition for the stability of the considered system, given in Proposition 3 in Section IV-A, and in the numerical approximation of the necessary and sufficient condition (31) developed in section IV-C.

\section{A. Integral Equations}

Following the method of characteristics, and integrating through the characteristic lines emerging from the boundaries (14) where the kernels are defined, we find that $L^{i j}, i, j \in$ $\{1,2\}$ verify the following integral equations

$$
\begin{aligned}
L^{11}(x, y)= & -\frac{\sigma_{1}}{q} \frac{r_{1}^{2}}{r}+r_{1} \sigma_{1} \int_{0}^{y} L^{21}(\xi+x-y, \xi) \mathrm{d} \xi \\
& +\frac{\sigma_{1}}{q} \frac{r_{1}}{r_{2}} \int_{0}^{(x-y) \frac{r_{1} r_{2}}{r}} L^{22}\left(\frac{\xi}{r_{1}}\right. \\
& \left.+(x-y) \frac{r_{1}}{r},-\frac{\xi}{r_{2}}+(x-y) \frac{r_{1}}{r}\right) \mathrm{d} \xi, \\
L^{12}(x, y)= & -\sigma_{1} \frac{r_{1} r_{2}}{r} \\
& +\sigma_{1} \int_{0}^{(x-y) \frac{r_{1} r_{2}}{r}} L^{22}\left(\frac{\xi}{r_{1}}\right. \\
& \left.+x \frac{r_{1}}{r}+y \frac{r_{2}}{r},-\frac{\xi}{r_{2}}+x \frac{r_{1}}{r}+y \frac{r_{2}}{r}\right) \mathrm{d} \xi,
\end{aligned}
$$

$$
\begin{aligned}
L^{21}(x, y)= & \sigma_{2} \frac{r_{1} r_{2}}{r}-\sigma_{2} \int_{0}^{(x-y) \frac{r_{1} r_{2}}{r}} L^{11}\left(\frac{\xi}{r_{2}}\right. \\
& \left.+x \frac{r_{2}}{r}+y \frac{r_{1}}{r},-\frac{\xi}{r_{1}}+x \frac{r_{2}}{r}+y \frac{r_{1}}{r}\right) \mathrm{d} \xi, \\
L^{22}(x, y)= & \sigma_{2} q \frac{r_{2}^{2}}{r}-r_{2} \sigma_{2} \int_{0}^{y} L^{12}(\xi+x-y, \xi) \mathrm{d} \xi \\
& -\sigma_{2} q \frac{r_{2}}{r_{1}} \int_{0}^{(x-y) \frac{r_{1} r_{2}}{r}} L^{11}\left(\frac{\xi}{r_{2}}\right. \\
& \left.+(x-y) \frac{r_{2}}{r},-\frac{\xi}{r_{1}}+(x-y) \frac{r_{2}}{r}\right) \mathrm{d} \xi .
\end{aligned}
$$

A general form of these integral equations can be found in [10]. Let us write equations (54) in a compact matrix form as

$$
L(x, y)=L_{0}+A[L](x, y),
$$

with $L(x, y) \doteq\left[\begin{array}{l}L^{11}(x, y) \\ L^{12}(x, y) \\ L^{21}(x, y) \\ L^{22}(x, y)\end{array}\right], L_{0} \doteq\left[\begin{array}{c}-\frac{\sigma_{1}}{q} \frac{r_{1}^{2}}{r} \\ -\sigma_{1} \frac{r 1}{r} \\ \sigma_{2} \frac{r_{1} r_{2}}{r} \\ \sigma_{2} q \frac{r_{2}^{2}}{r}\end{array}\right]$.

$A$ is a linear integral operator defined as

$$
\begin{aligned}
& A[L] \\
& \doteq\left[\begin{array}{cccc}
0 & 0 & r_{1} \sigma_{1} A_{1} & \frac{\sigma_{1}}{q} \frac{r_{1}}{r_{2}} A_{2} \\
0 & 0 & 0 & \sigma_{1} A_{3} \\
-\sigma_{2} A_{4} & 0 & 0 & 0 \\
-\sigma_{2} q \frac{r_{2}}{r_{1}} A_{5} & -r_{2} \sigma_{2} A_{1} & 0 & 0
\end{array}\right]\left[\begin{array}{c}
L^{11} \\
L^{12} \\
L^{21} \\
L^{22}
\end{array}\right],
\end{aligned}
$$

with

$$
\begin{aligned}
A_{1}\left[L^{i j}(\cdot, \cdot)\right](x, y) \doteq & \int_{0}^{y} L^{i j}(\xi+x-y, \xi) \mathrm{d} \xi, \\
A_{2}\left[L^{22}(\cdot, \cdot)\right](x, y) \doteq & \int_{0}^{(x-y) \frac{r_{1} r_{2}}{r}} L^{22}\left(\frac{\xi}{r_{1}}+(x-y) \frac{r_{1}}{r},\right. \\
& \left.-\frac{\xi}{r_{2}}+(x-y) \frac{r_{1}}{r}\right) \mathrm{d} \xi, \\
A_{3}\left[L^{22}(\cdot, \cdot)\right](x, y) \doteq & \int_{0}^{(x-y) \frac{r_{1} r_{2}}{r}} L^{22}\left(\frac{\xi}{r_{1}}+x \frac{r_{1}}{r}\right. \\
& \left.+y \frac{r_{2}}{r},-\frac{\xi}{r_{2}}+x \frac{r_{1}}{r}+y \frac{r_{2}}{r}\right) \mathrm{d} \xi, \\
A_{4}\left[L^{11}(\cdot, \cdot)\right](x, y) \doteq & \int_{0}^{(x-y) \frac{r_{1} r_{2}}{r}} L^{11}\left(\frac{\xi}{r_{2}}+x \frac{r_{2}}{r}\right. \\
& \left.+y \frac{r_{1}}{r},-\frac{\xi}{r_{1}}+x \frac{r_{2}}{r}+y \frac{r_{1}}{r}\right) \mathrm{d} \xi, \\
A_{5}\left[L^{11}(\cdot, \cdot)\right](x, y) \doteq & \int_{0}^{(x-y) \frac{r_{1} r_{2}}{r}} L^{11}\left(\frac{\xi}{r_{2}}+(x-y) \frac{r_{2}}{r},\right. \\
& \left.-\frac{\xi}{r_{1}}+(x-y) \frac{r_{2}}{r}\right) \mathrm{d} \xi .
\end{aligned}
$$




\section{B. Successive approximation and power series expressions}

The solution of the integral equation (55) will be generated using the method of successive approximations (see, for instance, [50] and [51]) as

$$
L(x, y)=\sum_{n=0}^{\infty} A^{n}\left[L_{0}\right](x, y),
$$

with $A^{0}\left[L_{0}\right]=L_{0}$. We denote $L_{k}^{i j}(x, y)=A^{k}\left[L_{0}\right](x, y), k \in$ $\mathbb{N}$. We denote as well $\gamma(x, y) \stackrel{\doteq}{=} \sigma_{1} \sigma_{2}(x-y)\left(r_{2} x+r_{1} y\right)$ and $\bar{\gamma}(x, y) \doteq \sigma_{1} \sigma_{2}(x-y)\left(r_{1} x+r_{2} y\right)$. In order to get the general term of the power series satisfied by each $L^{i j}(x, y)$ as in (58), the first few terms $\left(L_{k}^{i j}(x, y), k=1 \cdots 5\right)$ have been expressed. The patterns were then obtained by induction. Adding the consecutive terms of each $L^{i j}, i, j \in\{1,2\}$, we get the following power series expressions:

$$
\begin{aligned}
& L^{11}(x, y)=-\frac{r_{1}^{2}}{r} \frac{\sigma_{1}}{q} \sum_{m=0}^{\infty} \frac{(-1)^{m}}{m !^{2}}\left[\frac{r_{1}^{2} r_{2}}{r^{2}} \gamma(x, y)\right]^{m} \\
& +\frac{r_{1}^{2} r_{2}}{r^{2}} \sigma_{1} \sigma_{2}\left(r_{2} x+r_{1} y\right) \\
& \times \sum_{m=0}^{\infty} \frac{(-1)^{m}}{m !(m+1) !}\left[\frac{r_{1}^{2} r_{2}}{r^{2}} \gamma(x, y)\right]^{m}, \\
& L^{12}(x, y)=-\frac{r_{1} r_{2}}{r} \sigma_{1} \sum_{m=0}^{\infty} \frac{(-1)^{m}}{m !^{2}}\left[\frac{r_{1} r_{2}^{2}}{r^{2}} \bar{\gamma}(x, y)\right]^{m} \\
& +q \frac{r_{1} r_{2}^{3}}{r^{2}} \sigma_{1} \sigma_{2}(x-y) \\
& \times \sum_{m=0}^{\infty} \frac{(-1)^{m}}{m !(m+1) !}\left[\frac{r_{1} r_{2}^{2}}{r^{2}} \bar{\gamma}(x, y)\right]^{m}, \\
& L^{21}(x, y)=\frac{r_{1} r_{2}}{r} \sigma_{2} \sum_{m=0}^{\infty} \frac{(-1)^{m}}{m !^{2}}\left[\frac{r_{1}^{2} r_{2}}{r^{2}} \gamma(x, y)\right]^{m} \\
& +\frac{r_{1}^{3} r_{2}}{r^{2}} \frac{\sigma_{1} \sigma_{2}}{q}(x-y) \\
& \times \sum_{m=0}^{\infty} \frac{(-1)^{m}}{m !(m+1) !}\left[\frac{r_{1}^{2} r_{2}}{r^{2}} \gamma(x, y)\right]^{m}, \\
& L^{22}(x, y)=q \frac{r_{2}^{2}}{r} \sigma_{2} \sum_{m=0}^{\infty} \frac{(-1)^{m}}{m !^{2}}\left[\frac{r_{1} r_{2}^{2}}{r^{2}} \bar{\gamma}(x, y)\right]^{m} \\
& +\frac{r_{1} r_{2}^{2}}{r^{2}} \sigma_{1} \sigma_{2}\left(r_{1} x+r_{2} y\right) \\
& \times \sum_{m=0}^{\infty} \frac{(-1)^{m}}{m !(m+1) !}\left[\frac{r_{1} r_{2}^{2}}{r^{2}} \bar{\gamma}(x, y)\right]^{m} .
\end{aligned}
$$

$$
\begin{aligned}
& L^{11}(x, y)=-\frac{r_{1}^{2}}{r} \frac{\sigma_{1}}{q} J_{0}\left(\frac{2 r_{1} \sqrt{r_{2}}}{r} \sqrt{\gamma(x, y)}\right) \\
& +\frac{r_{1} \sqrt{r_{2}}}{r} \sqrt{\frac{\sigma_{1} \sigma_{2}\left(r_{2} x+r_{1} y\right)}{x-y}} \\
& \times J_{1}\left(\frac{2 r_{1} \sqrt{r_{2}}}{r} \sqrt{\gamma(x, y)}\right), \\
& L^{12}(x, y)=-\frac{r_{1} r_{2}}{r} \sigma_{1} J_{0}\left(\frac{2 \sqrt{r_{1}} r_{2}}{r} \sqrt{\bar{\gamma}(x, y)}\right) \\
& +q \frac{\sqrt{r_{1}} r_{2}^{2}}{r} \sqrt{\frac{\sigma_{1} \sigma_{2}(x-y)}{r_{1} x+r_{2} y}} \\
& \times J_{1}\left(\frac{2 \sqrt{r_{1}} r_{2}}{r} \sqrt{\bar{\gamma}(x, y)}\right), \\
& L^{21}(x, y)=\frac{r_{1} r_{2}}{r} \sigma_{2} J_{0}\left(\frac{2 r_{1} \sqrt{r_{2}}}{r} \sqrt{\gamma(x, y)}\right) \\
& +\frac{r_{1}^{2} \sqrt{r_{2}}}{q r} \sqrt{\frac{\sigma_{1} \sigma_{2}(x-y)}{r_{2} x+r_{1} y}} \\
& \times J_{1}\left(\frac{2 r_{1} \sqrt{r_{2}}}{r} \sqrt{\gamma(x, y)}\right), \\
& L^{22}(x, y)=q \frac{r_{2}^{2}}{r} \sigma_{2} J_{0}\left(\frac{2 \sqrt{r_{1}} r_{2}}{r} \sqrt{\bar{\gamma}(x, y)}\right) \\
& +\frac{\sqrt{r_{1}} r_{2}}{r} \sqrt{\frac{\sigma_{1} \sigma_{2}\left(r_{1} x+r_{2} y\right)}{x-y}} \\
& \times J_{1}\left(\frac{2 \sqrt{r_{1}} r_{2}}{r} \sqrt{\bar{\gamma}(x, y)}\right) .
\end{aligned}
$$

Notice that for $x=y$, the above expressions are well defined and simplify to

$$
\begin{aligned}
& L^{11}(x, x)=-\frac{r_{1}^{2}}{r} \frac{\sigma_{1}}{q}+\frac{r_{1}^{2} r_{2}}{r} \sigma_{1} \sigma_{2} x \\
& L^{12}(x, x)=-\frac{r_{1} r_{2}}{r} \sigma_{1} \\
& L^{21}(x, x)=\frac{r_{1} r_{2}}{r} \sigma_{2} \\
& L^{22}(x, x)=q \frac{r_{2}^{2}}{r} \sigma_{2}+\frac{r_{1} r_{2}^{2}}{r} \sigma_{1} \sigma_{2} x .
\end{aligned}
$$

Since it can be problematic to numerically manipulate (60) around $x=y$, we can use some properties of the Bessel functions as [52]

\section{Explicit expressions}

Using the Bessel functions of the first kind $J_{0}$ and $J_{1}$ [52], we have that

$$
J_{n}(\xi)=\frac{\xi}{2 n}\left(J_{n-1}(\xi)+J_{n+1}(\xi)\right) \forall n \in \mathbb{Z} \text { and } \forall \xi \in \mathbb{R},
$$

to get 
(63)

(64)

(65)

(66)

$$
\begin{aligned}
L^{11}(x, y)= & {\left[-\frac{r_{1}^{2}}{r} \frac{\sigma_{1}}{q}+\frac{r_{1}^{2} r_{2}}{r^{2}} \sigma_{1} \sigma_{2}\left(r_{2} x+r_{1} y\right)\right] } \\
& \times J_{0}\left(\frac{2 r_{1} \sqrt{r_{2}}}{r} \sqrt{\gamma(x, y)}\right) \\
& +\frac{r_{1}^{2} r_{2}}{r^{2}} \sigma_{1} \sigma_{2}\left(r_{2} x+r_{1} y\right) \\
& \times J_{2}\left(\frac{2 r_{1} \sqrt{r_{2}}}{r} \sqrt{\gamma(x, y)}\right), \\
L^{12}(x, y)= & \left.-\frac{r_{1} r_{2}}{r} \sigma_{1}+q \frac{r_{1} r_{2}^{3}}{r^{2}} \sigma_{1} \sigma_{2}(x-y)\right] \\
& \times J_{0}\left(\frac{2 \sqrt{r_{1}} r_{2}}{r} \sqrt{\bar{\gamma}(x, y)}\right) \\
& +q \frac{r_{1} r_{2}^{3}}{r^{2}} \sigma_{1} \sigma_{2}(x-y) \\
& \times J_{2}\left(\frac{2 \sqrt{r_{1}} r_{2}}{r} \sqrt{\bar{\gamma}(x, y)}\right), \\
& +\frac{r_{1} r_{2}^{2}}{r^{2}} \sigma_{1} \sigma_{2}\left(r_{1} x+r_{2} y\right) \\
& \times J_{2}\left(\frac{2 \sqrt{r_{1}} r_{2}}{r} \sqrt{\bar{\gamma}(x, y)}\right) \\
L^{21}(x, y)= & \left.\frac{r_{1} r_{2}}{r} \sigma_{2}+\frac{r_{1}^{3} r_{2}}{r^{2}} \frac{\sigma_{1} \sigma_{2}}{q}(x-y)\right] \\
& \times J_{0}\left(\frac{2 r_{1} \sqrt{r_{2}}}{r} \sqrt{\gamma(x, y)}\right) \\
& +\frac{r_{1}^{3} r_{2}}{r^{2}} \frac{\sigma_{1} \sigma_{2}}{q}(x-y) \\
& \times J_{2}\left(\frac{2 r_{1} \sqrt{r_{2}}}{r} \sqrt{\gamma(x, y)}\right), \\
L^{22}(x, y)= & {\left[q \frac{r_{2}^{2}}{r} \sigma_{2}+\frac{r_{1} r_{2}^{2}}{r^{2}} \sigma_{1} \sigma_{2}\left(r_{1} x+r_{2} y\right)\right] } \\
& \times J_{0}\left(\frac{2 \sqrt{r_{1}} r_{2}}{r} \sqrt{\bar{\gamma}(x, y)}\right) \\
&
\end{aligned}
$$

Remark 3: In the case $\sigma_{1} \sigma_{2}<0$, it may be easier to use the modified Bessel functions of the first kind $I_{n}$, having the relation $J_{n}(i \xi)=i^{n} I_{n}(\xi), \forall n \in \mathbb{Z}, \forall \xi \in \mathbb{R}$ [52].

\section{APPENDIX B}

\section{EXPRESSION AND BOUNDS OF $N(\theta)$}

\section{A. Expression of $N(\theta)$}

The explicit criterion given in Proposition 3 is based on the expressions of $N(\theta)$ and the upper-bound of $|N(\theta)|, \theta \in[0, r]$, as function of the original system parameters $\left(\sigma_{1}, \sigma_{2}, r_{1}, r_{2}, \rho\right.$ and $q$ ). In the sequel, we find these expressions before differentiating $N(\theta)$ and upper-bound its absolute value.

For $\theta \in\left[0, r_{2}\right]$, evaluating (64) and (66) at $x=1$ and $y=$ $1-\frac{\theta}{r_{2}}$ yields

$$
\begin{aligned}
\frac{1}{r_{2}} L^{22}\left(1,1-\frac{\theta}{r_{2}}\right) & \\
= & {\left[q \frac{r_{2}}{r} \sigma_{2}+\frac{R}{r^{2}}(r-\theta)\right] J_{0}\left(\frac{2 \sqrt{R \theta(r-\theta)}}{r}\right) } \\
& +\frac{R}{r^{2}}(r-\theta) J_{2}\left(\frac{2 \sqrt{R \theta(r-\theta)}}{r}\right), \\
-\frac{\rho}{r_{2}} & L^{12}\left(1,1-\frac{\theta}{r_{2}}\right) \\
= & {\left[\rho \frac{r_{1}}{r} \sigma_{1}-\rho q \frac{R}{r^{2}} \theta\right] J_{0}\left(\frac{2 \sqrt{R \theta(r-\theta)}}{r}\right) } \\
& -\rho q \frac{R}{r^{2}} \theta J_{2}\left(\frac{2 \sqrt{R \theta(r-\theta)}}{r}\right),
\end{aligned}
$$

where $R$ is defined in (33). For $\theta \in\left(r_{2}, r\right]$, evaluating (63) and (65) at $x=1$ and $y=\frac{\theta-r_{2}}{r_{1}}$ yields

$$
\begin{aligned}
\frac{q}{r_{1}} L^{21}\left(1, \frac{\theta-r_{2}}{r_{1}}\right) & \\
= & {\left[q \frac{r_{2}}{r} \sigma_{2}+\frac{R}{r^{2}}(r-\theta)\right] J_{0}\left(\frac{2 \sqrt{R \theta(r-\theta)}}{r}\right) } \\
& +\frac{R}{r^{2}}(r-\theta) J_{2}\left(\frac{2 \sqrt{R \theta(r-\theta)}}{r}\right), \\
-\frac{\rho q}{r_{1}} L^{11}\left(1, \frac{\theta-r_{2}}{r_{1}}\right) & \\
= & {\left[\rho \frac{r_{1}}{r} \sigma_{1}-\rho q \frac{R}{r^{2}} \theta\right] J_{0}\left(\frac{2 \sqrt{R \theta(r-\theta)}}{r}\right) } \\
& -\rho q \frac{R}{r^{2}} \theta J_{2}\left(\frac{2 \sqrt{R \theta(r-\theta)}}{r}\right) .
\end{aligned}
$$

Plugging these expressions in (19) and (25) we obtain

$$
\begin{aligned}
N(\theta)= & \left(\frac{a}{r}+\frac{d(\theta)}{r^{2}}\right) J_{0}(2 \sqrt{h(\theta)}) \\
& +\frac{d(\theta)}{r^{2}} J_{2}(2 \sqrt{h(\theta)}), \quad \forall \theta \in[0, r],
\end{aligned}
$$

where we defined

$$
\begin{aligned}
& d(\theta) \doteq R[r-\theta(1+\rho q)], \\
& h(\theta) \doteq \frac{R}{r^{2}} \theta(r-\theta)
\end{aligned}
$$

and $a$ is defined in (32).

\section{B. Bounds of $|N(\theta)|$ and $\left|N^{\prime}(\theta)\right|$}

The following calculations will be valid for $\sigma_{1} \sigma_{2} \geq 0$. The case where $\sigma_{1} \sigma_{2}<0$ will be treated next. We find an upper bound of $|N(\theta)|$ using some properties of the Bessel functions of the first kind [52] such as

$$
\begin{aligned}
\left|J_{0}(2 \sqrt{h(\theta)})\right| & \leq 1, \quad \forall \theta \in[0, r] \\
\left|J_{0}(2 \sqrt{h(\theta)})+J_{2}(2 \sqrt{h(\theta)})\right| & \leq 1, \quad \forall \theta \in(0, r) .
\end{aligned}
$$


By remarking that $|d(\theta)| \leq|R| r$, we have

$$
|N(\theta)| \leq \frac{1}{r}(|a|+|R|) .
$$

Differentiating $N(\theta)$ we get

$$
\begin{aligned}
N^{\prime}(\theta)= & \frac{d^{\prime}(\theta)}{r^{2}}\left[J_{0}(2 \sqrt{h(\theta)})+J_{2}(2 \sqrt{h(\theta)})\right] \\
& +\left(\frac{a}{r}+\frac{d(\theta)}{r^{2}}\right) J_{0}^{\prime}(2 \sqrt{h(\theta)}) \frac{h^{\prime}(\theta)}{\sqrt{h(\theta)}} \\
& +\frac{d(\theta)}{r^{2}} J_{2}^{\prime}(2 \sqrt{h(\theta)}) \frac{h^{\prime}(\theta)}{\sqrt{h(\theta)}},
\end{aligned}
$$

with

$$
\begin{aligned}
d^{\prime}(\theta) & =-R(1+\rho q) \\
h^{\prime}(\theta) & =\frac{R}{r}\left(1-2 \frac{\theta}{r}\right) \\
J_{0}^{\prime}(2 \sqrt{h(\theta)}) & =\frac{1}{2}\left[J_{-1}(2 \sqrt{h(\theta)})-J_{1}(2 \sqrt{h(\theta)})\right] \\
& =-J_{1}(2 \sqrt{h(\theta)}), \\
J_{2}^{\prime}(2 \sqrt{h(\theta)}) & =\frac{1}{2}\left[J_{1}(2 \sqrt{h(\theta)})-J_{3}(2 \sqrt{h(\theta)})\right] .
\end{aligned}
$$

Plugging (74) in (73) and using (62) yields

$$
\begin{aligned}
N^{\prime}(\theta)= & -\frac{R}{r^{2}}\left[1+\rho q+\left(1-2 \frac{\theta}{r}\right)\left(a+\frac{1}{2} \frac{d(\theta)}{r}\right)\right] \\
& \times J_{0}(2 \sqrt{h(\theta)}) \\
& -\frac{R}{r^{2}}\left[1+\rho q+\left(1-2 \frac{\theta}{r}\right)\left(a+\frac{2}{3} \frac{d(\theta)}{r}\right)\right] \\
& \times J_{2}(2 \sqrt{h(\theta)}) \\
& -\frac{1}{6} \frac{R}{r^{2}} \frac{d(\theta)}{r}\left(1-2 \frac{\theta}{r}\right) J_{4}(2 \sqrt{h(\theta)}) .
\end{aligned}
$$

Using the inequalities verified by the Bessel functions of first kind: $\left|J_{0}(2 \sqrt{h(\theta)})+J_{2}(2 \sqrt{h(\theta)})\right| \leq 1$ and $\left|J_{0}(2 \sqrt{h(\theta)})+\frac{4}{3} J_{2}(2 \sqrt{h(\theta)})+\frac{1}{3} J_{4}(2 \sqrt{h(\theta)})\right| \leq 1$ and by remarking that $\left|1-2 \frac{\theta}{r}\right| \leq 1$, we can bound $\left|N^{\prime}(\theta)\right|$ as

$$
\left|N^{\prime}(\theta)\right| \leq \frac{|R|}{r^{2}}\left(1+\rho q+|a|+\frac{|R|}{2}\right) .
$$

We should note that when $\sigma_{1} \sigma_{2}<0,|N(\theta)|$ and $\left|N^{\prime}(\theta)\right|$ are bounded differently. Recalling (69) and according to Remark $3, N(\theta)$ can be written in this case as

$$
N(\theta)=\left(\frac{a}{r}+\frac{d(\theta)}{r^{2}}\right) I_{0}(2 \sqrt{\bar{h}(\theta)})-\frac{d(\theta)}{r^{2}} I_{2}(2 \sqrt{\bar{h}(\theta)}),
$$

where we have defined

$$
\bar{h}(\theta) \doteq|h(\theta)|=\frac{|R|}{r^{2}} \theta(r-\theta) .
$$

According to the property of the modified Bessel functions [52]: $\forall n \in \mathbb{Z}, I_{n}(\theta)-I_{n+2}(\theta) \geq 0, \forall \theta \geq 0$ and
$I_{n}(\cdot)-I_{n+2}(\cdot)$ is a strictly increasing function, $|N(\theta)|$ can be bounded as

$$
|N(\theta)| \leq \frac{|a|}{r} I_{0}(\sqrt{|R|})+\frac{|R|}{r}\left[I_{0}(\sqrt{|R|})-I_{2}(\sqrt{|R|})\right] .
$$

$N^{\prime}(\theta)$ in this case can be deduced from (75) by following Remark 3 as

$$
\begin{aligned}
N^{\prime}(\theta)= & -\frac{R}{r^{2}}\left[1+\rho q+\left(1-2 \frac{\theta}{r}\right)\left(a+\frac{1}{2} \frac{d(\theta)}{r}\right)\right] \\
& \times I_{0}(2 \sqrt{\bar{h}(\theta)}) \\
& +\frac{R}{r^{2}}\left[1+\rho q+\left(1-2 \frac{\theta}{r}\right)\left(a+\frac{2}{3} \frac{d(\theta)}{r}\right)\right] \\
& \times I_{2}(2 \sqrt{\bar{h}(\theta)}) \\
& -\frac{1}{6} \frac{R}{r^{2}} \frac{d(\theta)}{r}\left(1-2 \frac{\theta}{r}\right) I_{4}(2 \sqrt{\bar{h}(\theta)}) .
\end{aligned}
$$

$\left|N^{\prime}(\theta)\right|$ can be bounded using the modified Bessel functions as in (79)

$$
\begin{aligned}
& \left|N^{\prime}(\theta)\right| \leq \frac{|R|}{r^{2}}\left(1+\rho q+|a|+\frac{|R|}{2}\right)\left[I_{0}(\sqrt{|R|})-I_{2}(\sqrt{|R|})\right] \\
& +\frac{R^{2}}{6 r^{2}}\left[I_{2}(\sqrt{|R|})-I_{4}(\sqrt{|R|})\right] .
\end{aligned}
$$

We define $\nu$ and $\bar{\nu}$ as upper-bounds of $|N(\theta)|$ and $\left|N^{\prime}(\theta)\right|$ respectively, as function of the system parameters, deduced from (72), (76), (79) and (81):

$$
\begin{aligned}
& \nu=\left\{\begin{array}{l}
\frac{1}{r}(|a|+R) \\
\text { if } \quad \sigma_{1} \sigma_{2} \geq 0, \\
\frac{|a|}{r} I_{0}(\sqrt{|R|})+\frac{|R|}{r}\left[I_{0}(\sqrt{|R|})-I_{2}(\sqrt{|R|})\right] \\
\text { if } \quad \sigma_{1} \sigma_{2}<0,
\end{array}\right. \\
& =\left\{\begin{array}{l}
\frac{|R|}{r^{2}}\left(1+\rho q+|a|+\frac{|R|}{2}\right) \\
\text { if } \sigma_{1} \sigma_{2} \geq 0, \\
\frac{|R|}{r^{2}}\left(1+\rho q+|a|+\frac{|R|}{2}\right)\left[I_{0}(\sqrt{|R|})-I_{2}(\sqrt{|R|})\right] \\
+\frac{R^{2}}{6 r^{2}}\left[I_{2}(\sqrt{|R|})-I_{4}(\sqrt{|R|})\right] \\
\text { if } \sigma_{1} \sigma_{2}<0 .
\end{array}\right.
\end{aligned}
$$

The values of $\nu$ and $\bar{\nu}$ in (82) will be used in the construction of the algorithm in section IV-C. However, in order to be more precise, and give the integral term in the sufficient condi- 
tion (36) a less conservative approximation, we express $|d(\theta)|$ depending on the sign of $\rho q$ as

$$
|d(\theta)|=\left\{\begin{array}{l}
|R|[r-\theta(1+\rho q)] \mathbb{1}_{\left[0, \frac{r}{1+\rho q}\right]}(\theta) \\
+|R|[-r+\theta(1+\rho q)] \mathbb{1}_{\left(\frac{r}{1+\rho q}, r\right]}(\theta) \\
\text { if } \quad \rho q \geq 0 \\
|R|[r-\theta(1+\rho q)] \\
\text { if } \quad \rho q<0,
\end{array}\right.
$$

which yields the following upper-bound of $\int_{0}^{r}|N(\theta)| \mathrm{d} \theta$, using (69), (77) and (83)

$$
\begin{aligned}
& \int_{0}^{r}|N(\theta)| \mathrm{d} \theta \leq \\
& \left\{\begin{array}{l}
|a|+|R|\left(\frac{1}{1+|\rho q|}-\frac{1-|\rho q|}{2}\right) \\
\text { if } \quad \sigma_{1} \sigma_{2} \geq 0 \quad \text { and } \quad \rho q \geq 0, \\
|a|+|R| \frac{1+|\rho q|}{2} \\
\text { if } \quad \sigma_{1} \sigma_{2} \geq 0 \quad \text { and } \quad \rho q<0, \\
|a| I_{0}(\sqrt{|R|})+|R|\left(\frac{1}{1+|\rho q|}-\frac{1-|\rho q|}{2}\right) \\
\times\left[I_{0}(\sqrt{|R|})-I_{2}(\sqrt{|R|})\right] \\
\text { if } \quad \sigma_{1} \sigma_{2}<0 \quad \text { and } \quad \rho q \geq 0, \\
|a| I_{0}(\sqrt{|R|})+|R| \frac{1+|\rho q|}{2}\left[I_{0}(\sqrt{|R|})-I_{2}(\sqrt{|R|})\right] \\
\text { if } \quad \sigma_{1} \sigma_{2}<0 \quad \text { and } \quad \rho q<0 .
\end{array}\right.
\end{aligned}
$$

\section{REFERENCES}

[1] P. C. Magnusson, A. Weisshaar, V. K. Tripathi, and G. C. Alexander, Transmission lines and wave propagation. CRC Press, 2000.

[2] J. de Halleux, C. Prieur, J.-M. Coron, B. d'Andréa Novel, and G. Bastin, "Boundary feedback control in networks of open channels," Automatica, vol. 39, no. 8, pp. 1365-1376, 2003.

[3] D. Rager, R. Neumann, and H. Murrenhoff, "Simplified fluid transmission line model for pneumatic control applications," in Proc. 14th Scandinavian International Conference on Fluid Power (SICFP15). Tampere, Finland, 2015.

[4] M. Gugat and M. Dick, "Time-delayed boundary feedback stabilization of the isothermal Euler equations with friction," Math. Control Relat. Fields, vol. 1, no. 4, pp. 469-491, 2011.

[5] C.-Z. Xu and G. Sallet, "Exponential stability and transfer functions of processes governed by symmetric hyperbolic systems," ESAIM: Control, Optimisation and Calculus of Variations, vol. 7, pp. 421-442, 2002.

[6] O. M. Aamo, "Leak detection, size estimation and localization in pipe flows," IEEE Transactions on Automatic Control, vol. 61, no. 1, pp. 246-251, 2016.

[7] J.-M. Coron, B. d'Andrea Novel, and G. Bastin, "A strict Lyapunov function for boundary control of hyperbolic systems of conservation laws," IEEE Transactions on Automatic Control, vol. 52, no. 1, pp. 2 11, 2007.

[8] V. Dos Santos, G. Bastin, J.-M. Coron, and B. d'Andréa Novel, "Boundary control with integral action for hyperbolic systems of conservation laws: Stability and experiments," Automatica, vol. 44, no. 5, pp. 1310 1318,2008

[9] R. Vazquez, M. Krstic, and J.-M. Coron, "Backstepping boundary stabilization and state estimation of a $2 \times 2$ linear hyperbolic system," in Proceedings of the 50th IEEE Conference on Decision and Control and European Control Conference. IEEE, 2011, pp. 4937-4942.
[10] J.-M. Coron, R. Vazquez, M. Krstic, and G. Bastin, "Local exponential $H^{2}$ stabilization of a $2 \times 2$ quasilinear hyperbolic system using backstepping," SIAM Journal on Control and Optimization, vol. 51, no. 3, pp. 2005-2035, 2013.

[11] P.-O. Lamare and N. Bekiaris-Liberis, "Control of $2 \times 2$ linear hyperbolic systems: Backstepping-based trajectory generation and PI-based tracking," Systems \& Control Letters, vol. 86, pp. 24-33, 2015.

[12] G. Bastin and J.-M. Coron, Stability and boundary stabilization of 1$D$ hyperbolic systems, ser. Progress in Nonlinear Differential Equations and Their Applications Subseries in Control. Birkhäuser, 2016, vol. 88.

[13] J.-M. Coron, G. Bastin, and B. d'Andréa Novel, "Dissipative boundary conditions for one-dimensional nonlinear hyperbolic systems," SIAM Journal on Control and Optimization, vol. 47, no. 3, pp. 1460-1498, 2008.

[14] C. Prieur, A. Girard, and E. Witrant, "Lyapunov functions for switched linear hyperbolic systems," IFAC Proceedings Volumes, vol. 45, no. 9 , pp. 382-387, 2012.

[15] A. Tchousso, T. Besson, and C.-Z. Xu, "Exponential stability of distributed parameter systems governed by symmetric hyperbolic partial differential equations using Lyapunov's second method," ESAIM: Control, Optimisation and Calculus of Variations, vol. 15, no. 2, pp. 403425, 2009.

[16] M. Safi, L. Baudouin, and A. Seuret, "Tractable sufficient stability conditions for a system coupling linear transport and differential equations," Systems \& Control Letters, vol. 110, pp. 1-8, 2017.

[17] Y. Tang, C. Prieur, and A. Girard, "Tikhonov theorem for linear hyperbolic systems," Automatica, vol. 57, pp. 1-10, 2015.

[18] , "Singular perturbation approximation of linear hyperbolic systems of balance laws," IEEE Transactions on Automatic Control, vol. 61, no. 10, pp. 3031-3037, 2016.

[19] Y. Tang and G. Mazanti, "Stability analysis of coupled linear ODEhyperbolic PDE systems with two time scales," Automatica, vol. 85, pp. 386-396, 2017.

[20] Y. Tang, C. Prieur, and A. Girard, "Stability analysis of a singularly perturbed coupled ODE-PDE system," in Decision and Control (CDC), 2015 IEEE 54th Annual Conference on. IEEE, 2015, pp. 4591-4596.

[21] E. Cerpa and C. Prieur, "Effect of time scales on stability of coupled systems involving the wave equation," in IEEE Conf. on Dec. and Cont.(CDC'17), 2017.

[22] I. Karafyllis and M. Krstic, Input-to-State Stability for PDEs. Springer International Publishing, 2019.

[23] D. L. Russell, "Neutral FDE canonical representations of hyperbolic systems," The Journal of Integral Equations and Applications, pp. 129166, 1991.

[24] _ "Canonical forms and spectral determination for a class of hyperbolic distributed parameter control systems," Journal of Mathematical Analysis and Applications, vol. 62, no. 1, pp. 186-225, 1978.

[25] I. Karafyllis and M. Krstic, "On the relation of delay equations to first-order hyperbolic partial differential equations," ESAIM: Control, Optimisation and Calculus of Variations, vol. 20, no. 3, pp. 894-923, 2014.

[26] J. Auriol, F. Bribiesca-Argomedo, D. Bou Saba, M. Di Loreto, and F. Di Meglio, "Delay-robust stabilization of a hyperbolic PDE-ODE system," Automatica, vol. 95, pp. 494-502, 2018.

[27] S. Damak, M. Di Loreto, and S. Mondié, "Stability of linear continuoustime difference equations with distributed delay: Constructive exponential estimates," International Journal of Robust and Nonlinear Control, vol. 25, no. 17, pp. 3195-3209, 2015.

[28] D. Melchor-Aguilar, "Exponential stability of linear continuous time difference systems with multiple delays," Systems \& Control Letters, vol. 62 , no. 10 , pp. 811-818, 2013.

[29] J. K. Hale and S. M. V. Lunel, "Strong stabilization of neutral functional differential equations," IMA Journal of Mathematical Control and Information, vol. 19, no. 1_and_2, pp. 5-23, 2002.

[30] V. Y. Glizer, E. Fridman, and Y. Feigin, "A novel approach to exact slowfast decomposition of linear singularly perturbed systems with small delays," SIAM Journal on Control and Optimization, vol. 55, no. 1, pp. 236-274, 2017.

[31] R. E. Bellman and K. L. Cooke, "Differential-difference equations," 1963.

[32] O. Diekmann, S. A. Van Gils, S. M. Lunel, and H.-O. Walther, Delay equations: functional-, complex-, and nonlinear analysis. Springer Science \& Business Media, 2012, vol. 110.

[33] S.-I. Niculescu, "On delay-dependent stability under model transformations of some neutral linear systems," International Journal of Control, vol. 74, no. 6, pp. 609-617, 2001. 
[34] F. Di Meglio, R. Vazquez, and M. Krstic, "Stabilization of a linear hyperbolic system with one boundary controlled transport PDE coupled with n counterconvecting PDEs," in Decision and Control (CDC), 2012 IEEE 51st Annual Conference on. IEEE, 2012, pp. 2914-2919.

[35] L. Hu, F. Di Meglio, R. Vazquez, and M. Krstic, "Control of homodirectional and general heterodirectional linear coupled hyperbolic PDEs," IEEE Transactions on Automatic Control, vol. 61, no. 11, pp. 33013314, 2016.

[36] F. Di Meglio, F. Bribiesca-Argomedo, L. Hu, and M. Krstic, "Stabilization of coupled linear heterodirectional hyperbolic PDE-ODE systems," Automatica, vol. 87, pp. 281-289, 2018.

[37] J. Auriol and F. Di Meglio, "Minimum time control of heterodirectional linear coupled hyperbolic PDEs," Automatica, vol. 71, pp. 300-307, 2016.

[38] — - "Two-sided boundary stabilization of two linear hyperbolic PDEs in minimum time," in Decision and Control (CDC), 2016 IEEE 55th Conference on. IEEE, 2016, pp. 3118-3124.

[39] J. Auriol, U. J. F. Aarsnes, P. Martin, and F. Di Meglio, "Delay-robust control design for heterodirectional linear coupled hyperbolic PDEs," IEEE Transactions on Automatic Control, 2018.

[40] H. Ramirez, H. Zwart, Y. Le Gorrec, and A. Macchelli, "On backstepping boundary control for a class of linear port-hamiltonian systems," in Decision and Control (CDC), 2017 IEEE 56th Annual Conference on. IEEE, 2017, pp. 658-663.

[41] A. Macchelli, Y. Le Gorrec, and H. Ramírez, "Boundary control of distributed port-hamiltonian systems via generalised canonical transformations," in Decision and Control (CDC), 2017 IEEE 56th Annual Conference on. IEEE, 2017, pp. 70-75.

[42] R. Vazquez and M. Krstic, "Marcum Q-functions and explicit kernels for stabilization of $2 \times 2$ linear hyperbolic systems with constant coefficients," Systems \& Control Letters, vol. 68, pp. 33-42, 2014.

[43] M. Vidyasagar, "Input-output stability of a broad class of linear timeinvariant multivariable systems," SIAM Journal on Control, vol. 10, no. 1, pp. 203-209, 1972

[44] G. Bastin and J.-M. Coron, "On boundary feedback stabilization of non-uniform linear $2 \times 2$ hyperbolic systems over a bounded interval," Systems \& Control Letters, vol. 60, no. 11, pp. 900-906, 2011.

[45] R. Baker and D. Vakharia, "Input-output stability of linear time-invariant systems," IEEE Transactions on Automatic Control, vol. 15, no. 3, pp. 316-319, 1970.

[46] S. Gumussoy and W. Michiels, "A predictor-corrector type algorithm for the pseudospectral abscissa computation of time-delay systems," Automatica, vol. 46, no. 4, pp. 657-664, 2010.

[47] D. Avanessoff, A. Fioravanti, and C. Bonnet, "Yalta: a Matlab toolbox for the $\mathrm{H}_{\infty}$-stability analysis of classical and fractional systems with commensurate delays," IFAC Proceedings Volumes, vol. 46, no. 2, pp. 839-844, 2013.

[48] K. Engelborghs, T. Luzyanina, and D. Roose, "Numerical bifurcation analysis of delay differential equations using dde-biftool," ACM Transactions on Mathematical Software (TOMS), vol. 28, no. 1, pp. 1-21, 2002.

[49] W. Michiels and H. U. Ünal, "Evaluating and approximating FIR filters : An approach based on functions of matrices," IEEE Transactions on Automatic Control, vol. 60, no. 2, pp. 463-468, 2014.

[50] D. Carothers, W. Ingham, J. Liu, C. Lyons, J. Marafino, G. E. Parker, D. Pruett, J. Rudmin, J. S. Sochacki, D. Warne et al., "An overview of the modified Picard method," Department of Mathematics and Statistics, Physics, James Madison University, Harrisonburg, Report VA22807, 2004.

[51] M. Krstic and A. Smyshlyaev, "Backstepping boundary control for firstorder hyperbolic PDEs and application to systems with actuator and sensor delays," Systems \& Control Letters, vol. 57, no. 9, pp. 750-758, 2008.

[52] M. Kreh, "Bessel functions," Lecture Notes, Penn State-Göttingen Summer School on Number Theory, vol. 82, 2012.

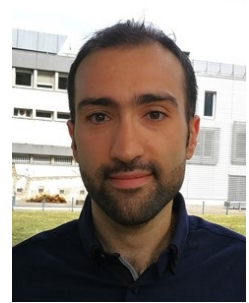

David Bou Saba received his mechanical engineering degree from the Lebanese University, Faculty of engineering, Roumieh, Lebanon, his M.Sc. degree in control systems from École Centrale of Lyon, France and his Ph.D. degree from Institut National des Sciences Appliquées of Lyon, France, under the direction of Federico Bribiesca-Argomedo. His research interests include analysis and control of hyperbolic PDEs.

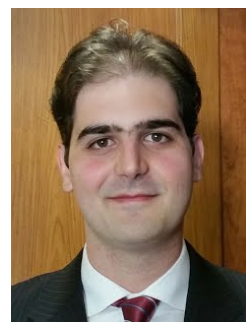

Federico Bribiesca-Argomedo received the B.Sc. degree in mechatronics engineering from the Tecnológico de Monterrey, Monterrey, Mexico, in 2009, the M.Sc. degree in control systems from Grenoble INP, Grenoble, France, in 2009, and the Ph.D. degree in control systems from the GIPSA-Laboratory, Grenoble University, Grenoble. He held a postdoctoral position with the Department of Mechanical and Aerospace Engineering, University of California at San Diego, CA, USA. He is currently an Associate Professor with the Department of Mechanical Engineering, Institut national des sciences appliquées de Lyon, Lyon, France, attached to Ampère Laboratory. Research interests include control of hyperbolic and parabolic partial differential equations and nonlinear control theory. Past and current applications include tokamak safety factor profiles, electrochemical models of $\mathrm{Li}$-ion batteries and energy distribution networks.

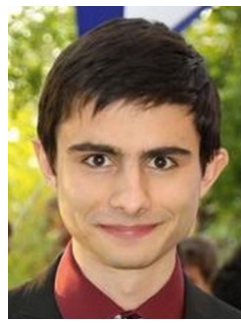

Jean Auriol received his Master degree in civil engineering in 2015 (major: applied maths) in MINES ParisTech, part of PSL Research University. He started the same year his $\mathrm{PhD}$ at Centre Automatique et Systèmes of MINES ParisTech, part of the same university, under the direction of Florent $\mathrm{Di}$ Meglio. His PhD subject deals with robust control, observability and estimation design of hyperbolic Partial Differential Equations using a backstepping approach.

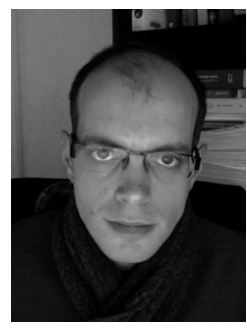

Michael Di Loreto obtained his Ph.D degree in control theory from Université de Nantes (France) in 2006. Since 2007, he is an associate professor at Laboratoire Ampère, INSA de Lyon, France. His main research interests are in time-delay systems, distributed parameter systems and linear control theory.

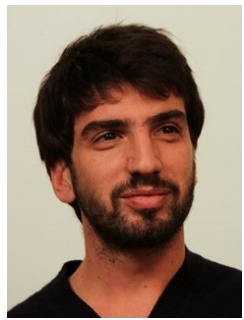

Florent Di Meglio is tenured professor at the Centre Automatique et Systèmes of MINES ParisTech, part of PSL Research University. He received his Ph.D. from the same university in Mathematics and Control in 2011, and was a Posdoctoral Researcher at UC San Diego from 2011 to 2012. His current research interests include control and estimation design for hyperbolic PDEs, with application to process control, most notably multiphase flow control and oil drilling. 